\title{
QUASI-INVARIANCE OF LOW REGULARITY GAUSSIAN MEASURES UNDER THE GAUGE MAP OF THE PERIODIC DERIVATIVE NLS
}

\author{
GIUSEPPE GENOVESE, RENATO LUCÀ, AND NIKOLAY TZVETKOV
}

\begin{abstract}
The periodic DNLS gauge is an anticipative map with singular generator which revealed crucial in the study of the periodic derivative NLS. We prove quasi-invariance of the Gaussian measure on $L^{2}(\mathbb{T})$ with covariance $\left[1+(-\Delta)^{s}\right]^{-1}$ under these transformations for any $s>\frac{1}{2}$. This extends previous achievements by Nahmod, Ray-Bellet, Sheffield and Staffilani (2011) and Genovese, Lucà and Valeri (2018), who proved the result for integer values of the regularity parameter $s$.
\end{abstract}

There may be differences between this version and the published version. You are advised to consult the publisher's version:

G. Genovese, R. Lucà and N. Tzvetkov, Quasi-invariance of low regularity Gaussian measures under the gauge map of the periodic derivative NLS, Journal of Functional Analysis Volume 282, Issue 1, 1 January 2022, https://doi.org/10.1016/j.jfa.2021.109263.

\section{INTRODUCTION}

Let $\mathbb{T}:=\mathbb{R} / 2 \pi \mathbb{Z}$ and $\alpha \in \mathbb{R}$. We introduce $\mathscr{G}_{\alpha}: L^{2}(\mathbb{T}) \rightarrow L^{2}(\mathbb{T})$ to be defined by

$$
\mathscr{G}_{\alpha}(u)(x):=e^{i \alpha \mathcal{I}[u(x)]} u(x),
$$

where

$$
\mathcal{I}[u(x)]:=\frac{1}{2 \pi} \int_{0}^{2 \pi} d \theta \int_{\theta}^{x}\left(|u(y)|^{2}-\frac{\|u\|_{L^{2}(\mathbb{T})}^{2}}{2 \pi}\right) d y .
$$

To get some insight, $\mathcal{I}[u(x)]$ can be though of as the unique periodic primitive of $|u|^{2}$ with zero average, therefore we can formally write

$$
\mathcal{I}[u(x)]=\partial_{x}^{-1}\left(|u|^{2}-\frac{1}{2 \pi}\|u\|_{L^{2}}^{2}\right) .
$$

We will much exploit this intuition in the sequel.

An alternative formulation of (1.1) is through the initial value problem

$$
\frac{d}{d \alpha} \mathscr{G}_{\alpha} u=i \mathcal{I}\left[\mathscr{G}_{\alpha} u\right] \mathscr{G}_{\alpha} u, \quad \mathscr{G}_{0} u=u .
$$

The map $\alpha \rightarrow \mathscr{G}_{\alpha}$ is a one parameter group of transformations of $L^{2}(\mathbb{T})$, in fact

$$
\mathscr{G}_{0}=\mathrm{Id} \quad \text { and } \quad \mathscr{G}_{\alpha_{1}} \circ \mathscr{G}_{\alpha_{2}}=\mathscr{G}_{\alpha_{1}+\alpha_{2}}, \quad \text { for any } \alpha_{1}, \alpha_{2} \in \mathbb{R} .
$$

This gauge was introduced in the periodic setting in [16] in the context of the derivative nonlinear Schödinger equation (DNLS). It has been conveniently used in different contexts regarding the DNLS: just to mention few examples, the study of the local well-posedness at low regularity is based on the use of such a gauge transformation $[16,15,8]$ and it revealed to be crucial also in the

Date: November 8, 2021

2000 Mathematics Subject Classification. 60G22, 35Q55, 37A05.

Key words and phrases. Gaussian measures, anticipative transformations. 
proof of the invariance of the Gibbs measures associated with the integrals of motions of DNLS $[22,12]$.

In this paper we investigate the way these maps transform the Gaussian measure on $L^{2}(\mathbb{T})$ with covariance operator $\left[1+(-\Delta)^{s}\right]^{-1}$ for $s>\frac{1}{2}$. Thanks to separability and the isomorphism between $\mathbb{C}^{2 N+1}$ and

$$
E_{N}:=\operatorname{span}_{\mathbb{C}}\left\{e^{i n x}:|n| \leqslant N\right\}
$$

the space $L^{2}(\mathbb{T})$ inherits the measurable-space structure by a standard limit procedure and we will denote by $\mathscr{B}\left(L^{2}(\mathbb{T})\right)$ the Borel $\sigma$-algebra on $L^{2}(\mathbb{T})$. We denote by $\gamma_{s}$ the Gaussian measure on $\mathscr{B}\left(L^{2}(\mathbb{T})\right)$ induced by the map

$$
\omega \longmapsto \sum_{n \in \mathbb{Z}} \frac{g_{n}(\omega)}{\left(1+|n|^{2 s}\right)^{\frac{1}{2}}} e^{i n x},
$$

where $\left\{g_{n}(\omega)\right\}_{n \in \mathbb{Z}}$ are independent, identically distributed complex centred Gaussian random variables with unitary variance. For any $s \in \mathbb{R}$ the triple $\left(L^{2}(\mathbb{T}), \mathscr{B}\left(L^{2}(\mathbb{T})\right), \gamma_{s}\right)$ is a Gaussian probability space satisfying the concentration properties:

$$
\gamma_{s}\left(\bigcap_{s^{\prime}<s-\frac{1}{2}} H^{s^{\prime}}(\mathbb{T})\right)=1, \quad \gamma_{s}\left(H^{s-\frac{1}{2}}(\mathbb{T})\right)=0 .
$$

The $L^{p}$ spaces associated to $\gamma_{s}$ are denoted by $L^{p}\left(\gamma_{s}\right)$. For more details about this construction we refer for instance to [19].

Our main result is the quasi-invariance under the group $\left\{\mathscr{G}_{\alpha}\right\}_{\alpha \in \mathbb{R}}$ of $\gamma_{s}$ restricted to a ball in $L^{2}(\mathbb{T})$, of arbitrary size, defined by $B(R):=\left\{u:\|u\|_{L^{2}(\mathbb{T})} \leqslant R\right\}$ for all $s>\frac{1}{2}$. Henceforth we set for brevity

$$
\tilde{\gamma}_{s}(A):=\gamma_{s}\left(A \cap\left\{\|u\|_{L^{2}} \leqslant R\right\}\right), \quad A \in \mathscr{B}\left(L^{2}(\mathbb{T})\right) .
$$

Theorem 1.1. Let $s>\frac{1}{2}, R>0$. Then for every $\alpha \in \mathbb{R}$ there is $p_{0}>1$ and $\rho_{\alpha} \in L^{p}\left(\tilde{\gamma}_{s}\right)$ for all $p \in\left[1, p_{0}\right)$ such that

$$
\left(\tilde{\gamma}_{s} \circ \mathscr{G}_{\alpha}\right)(A)=\int_{A} \rho_{\alpha}(u) \tilde{\gamma}_{s}(d u), \quad A \in \mathscr{B}\left(L^{2}(\mathbb{T})\right)
$$

The restriction of the measure to a ball $B(R)$ of $L^{2}$ is possible as $\mathscr{G}_{\alpha}$ leaves invariant the $L^{2}(\mathbb{T})$ norm for all $\alpha$. It is worthy to remark that, unlike all the other works on the subject [31, 23, 11, $12,3]$, we are not imposing any smallness assumption on $R$. This observation may be useful in the attempt of proving probabilistc global well-posedness for DNLS without imposing any smallness assumption on the $L^{2}$ norm. Remarkable results in this direction are [20, 17] and [1] where the authors prove that DNLS is globally well-posed on the real line $\mathbb{R}$ in weighted and in translation invariant Sobolev spaces, respectively. The well-posedness in the periodic setting and large $L^{2}$ norm remains a challenging open problem.

The transformation of Gaussian measures have been intensively studied since long, starting from the fundamental theorem of Cameron-Martin [4] for shift maps. The Cameron-Martin theorem was then extended in two non-overlapping directions, by Girsanov [13] (for non-anticipative maps) and by Kuo [18] and Ramer [29] (for anticipative maps). Nowadays these results are well established and the lay at the very basis of the development of stochastic calculus, but they have been achieved primarily by a functional analytic approach, exploiting the properties of the generator of the transformation, which needs to be at least Hilbert-Schmidt for anticipative maps. Further developments have been achieved by means of Malliavin calculus [5, 6, 21, 37], essentially identifying more general classes of maps allowing quasi-invariance of Gaussian measures in functional spaces.

The problem witnessed recently a resurgence of interest mainly concerning the evolution of the Brownian motion (or related processes) along the flow of dispersive PDEs [33, 24, 25, 26, 27, 28, 14, $30,10,7]$. A new analytic approach was introduced for flows of dispersive nonlinear equations in 
[33]. The argument (inspired by the previous works [34, 35, 36, 9]), exploits directly the properties of the flow of the PDE under consideration. However, this technique does not provide an explicit approximation of the density of the infinite dimensional change of coordinates induced by the flow.

Given this framework, the DNLS gauge transformations $\mathscr{G}_{\alpha}$ represent an interesting mathematical challenge, as they are anticipative maps whose generator is not Hilbert-Schmidt. Therefore none of the classical results [29, 13, 5, 6] applies. Nonetheless we can successfully exploit the method of [33] and we show in this paper that we can push it to deal with regularity up to $H^{r}(\mathbb{T})$ for any $r>0$, corresponding to Gaussian measures $\gamma_{s}$ with $s>\frac{1}{2}$. Our result extends (and improves, getting rid of the small $L^{2}$ norm restriction) the earlier analysis of [23, 12] valid for integer $s \geqslant 1$. It will become clear from our analysis that the restriction $s>\frac{1}{2}$ is most likely optimal. In particular for lower values of $s$ the support of $\gamma_{s}$ is no longer on classical functions and the extension of our result to such values of $s$ would certainly require some renormalisation procedure.

A special mention is deserved by the case $s=1$, addressed in [23] by the following nice probabilistic argument. The typical trajectories of $\gamma_{1}$ are complex Brownian bridges, for which modulus and phase are conditionally independent after a time-change. Since the gauge acts in fact as a modulus-dependent phase-shift, conditionally on the modulus Cameron-Martin theorem applies. In this way the authors were able not only to prove quasi-invariance but also the precise change of variable formula via Cameron-Martin theorem. Unfortunately this trick is very much based on the specific properties of the complex Brownian bridge and seems to be difficult to reproduce for general Gaussian measures. We stress that the small mass restriction here emerges by the so-called Novikov condition, which amounts to require uniform integrability of the density of the change of variables. To ensure this property the authors rely on the analysis of [31] of the Gibbs measure of the derivative NLS, as the leading order terms in the exponent of the density are the same.

Even though we do not attack the problem directly, our work gives a strong indication that the change of variable formula established in [23] does not require any condition on the mass. Otherwise the problem of determining the precise densities given by the gauge map is still open for $s \neq 1$. Let us point out that (with the notable exception of [7]) most of the works, appeared recently on the subject in the context of dispersive PDEs, cannot specify the Radon-Nykodim derivative by means of a suitable approximation procedure.

The low fractional regularity brings some new challenges. The main problem is to find a good replacement to the classical integration by parts formula (or equivalently in our case Leibniz formula) valid for fractional derivatives. Indeed the explicit representation of the variation of the Sobolev norm for integral regularity given in [12, Lemma 2.9] was obtained by a direct exploit of the Leibniz rule and does not easily generalise to fractional regularity. Therefore we have to base our analysis on a less transparent representation in terms of Fourier coefficients, which is not evidently of similar form. Indeed the gauge map can be written as identity plus smoothing only for high frequencies, but the low frequency contribution is hard to bound. Therefore we have to isolate the low frequency term and operate on it a fractional integration by parts in order to profit by a convenient cancellation given by the imaginary part as in the DNSL integrals of motion (the same kind of difficulty is solved in [30] with similar methods). In fact, as already remarked in [12], the terms appearing from the transformation along the flow of $\mathscr{G}_{\alpha}$ of the Sobolev norms are of the same type of the DNLS energies. Therefore, even though equation (1.3) is much easier than DNLS, therefore the analytical difficulties are less challenging, from the probabilistic viewpoint to study the transformed Sobolev norm via $\left\{\mathscr{G}_{\alpha}\right\}_{\alpha \in \mathbb{R}}$ or the energies of DNLS is mostly equivalent. This constitutes a crucial point of this work as, albeit the flow is very regular, the Sobolev norms are hard to bound within the support of the Gaussian measure $\gamma_{s}$.

The paper is organised as follows. In Section 2 a suitable approximation of the gauge map is presented and some first properties are stated. The most important being that this approximations behave well with the finite dimensional Lebesgue measures in the frequency space (see Section 4) and that the approximating gauge flow and the true gauge flow are asymptotically close. In 
Section 3 we present the main argument of the proof, which is essentially the adaptation from [33] to our case, leaving most of the specifics to the subsequent sections: in Section 4 we study the behaviour of the Jacobian determinant; in Section 5 and Section 6 we show that the derivative of the Sobolev norm along the flow computed in zero is a sub-exponential random variable w.r.t. $\gamma_{s}$ restricted to a ball of $L^{2}$. This is the most technical part of the paper. First in Section 5 we show the quantity of interest to converge in $L^{2}\left(\gamma_{s}\right)$ and then in Section 6 we employ the argument of [2] to show sub-exponential behaviour. In both sections we need to separate small and high frequencies as explained before. The splitting differs slightly in the two sections, but is similar in spirit.

Notations. Throughout we denote by $u(n)$ the $n$-th Fourier coefficient of $u: \mathbb{T} \rightarrow \mathbb{C} . E_{s}[\cdot]$ the expectation value w.r.t. $\gamma_{s}$. We define the fractional derivative of order $s$ as

$$
u^{(s)}(n)=|n|^{s} u(n) .
$$

We use the following definition for the fractional Sobolev seminorm

$$
\|u\|_{\dot{H}^{s}}^{2}=\sum_{n \in \mathbb{Z}, n \neq 0}|n|^{2 s}|u(n)|^{2}
$$

and we define the fractional Sobolev norm as

$$
\|u\|_{H^{s}}^{2}=\|u\|_{L^{2}}^{2}+\|u\|_{\dot{H}^{s}}^{2} .
$$

We have by Plancherel

$$
\|u\|_{\dot{H}^{s}}^{2}=\int\left|u^{(s)}\right|^{2} .
$$

We also have $\|\cdot\|_{H^{0}(\mathbb{T})} \simeq\|\cdot\|_{L^{2}(\mathbb{T})}$. Given $R>0$, we denote with $B(R)$ the ball of center zero and radius $R$ in the $L^{2}(\mathbb{T})$-topology. We set

$$
\tilde{\gamma}_{s}(A):=E_{s}\left[1_{B(R) \cap A}\right] .
$$

Let $P_{N}$ be the the projection on the first $N$ Fourier modes

$$
P_{N} \sum_{n \in \mathbb{Z}} e^{i n x} c_{n}:=\sum_{|n| \leqslant N} e^{i n x} c_{n} .
$$

We define

$$
\tilde{\gamma}_{s, N}(A):=E_{s}\left[1_{\left\{\left\|P_{N} u\right\|_{L^{2}} \leqslant R\right\} \cap A}\right]
$$

for any measurable $A$. Note that $R$ is always implicit in the definition of $\tilde{\gamma}_{s}$ and $\tilde{\gamma}_{s, N}$. For $j \in \mathbb{N}$ the Littlewood-Paley projector is denoted by $\Delta_{j}:=P_{2^{j}}-P_{2^{j-1}}$; we write $|n| \simeq 2^{j}$ to shorten $2^{j-1}<|n| \leqslant 2^{j}$ for $j \in \mathbb{N}$, while for $j=0|n| \simeq 1$ shortens $|n| \leqslant 1$. We write $X \lesssim Y$ to denote that $X \leqslant C Y$ for some positive constant $C$ independent on $X, Y$.

We denote by $E_{N}^{\perp}$ the orthogonal complement of $E_{N}$ in the topology of $L^{2}(\mathbb{T})$. Letting $\gamma_{s}^{\perp, N}$ the measure induced on $E_{N}^{\perp}$ by the map

$$
\omega \longmapsto \sum_{|n|>N} \frac{g_{n}(\omega)}{\left(1+|n|^{2 s}\right)^{\frac{1}{2}}} e^{i n x},
$$

the measure $\gamma_{s}$ factorises over $E_{N} \times E_{N}^{\perp}$ as

$$
\gamma_{s}(d u):=\frac{1}{Z_{N}} e^{-\frac{1}{2}\left\|P_{N} u\right\|_{H^{s}}^{2}} L_{N}\left(d P_{N} u\right) \gamma_{s}^{\perp, N}\left(d P_{>N} u\right)
$$

where $L_{N}$ is the Lebesgue measure induced on $E_{N}$ by the isomorphism between $\mathbb{R}^{2(2 N+1)}$ and $E_{N}$ and $Z_{N}$ is a renormalisation factor. 
We will use the Bernstein inequality for estimating tail probabilities in the following form. Let $X_{1}, \ldots, X_{N}$ i.i.d. sub-exponential component of $X \in \mathbb{R}^{N}$ and $a \in \mathbb{R}^{N}$. Then there are $c, C>0$ such that

$$
P\left(\left|\sum_{i=1}^{N} a_{i} X_{i}\right| \geqslant t\right) \leqslant \exp \left(-c \min \left(\frac{t}{\max _{i}\left|a_{i}\right|}, \frac{t^{2}}{\|a\|_{2}^{2}}\right)\right) .
$$

Acknowledgements. R. Lucà is supported by the Basque Government under program BCAMBERC 2018-2021, by the Spanish Ministry of Science, Innovation and Universities under the BCAM Severo Ochoa accreditation SEV-2017-0718 and by IHAIP project PGC2018-094528-B-I00 (AEI/FEDER, UE). N. Tzvetkov is supported by ANR grant ODA (ANR-18-CE40-0020-01).

\section{Approximated flow}

Let $P_{N}$ be the the projection on the first $N$ Fourier modes (see (1.11)). Given $N \in \mathbb{N}$ we define an approximation $\mathscr{G}_{\alpha}^{N}: L^{2}(\mathbb{T}) \rightarrow L^{2}(\mathbb{T})$ of $\mathscr{G}_{\alpha}$ using the truncated system (compare with (1.3))

$$
\frac{d}{d \alpha} \mathscr{G}_{\alpha}^{N}(u)=i P_{N}\left(\mathcal{I}\left[P_{N} \mathscr{G}_{\alpha}^{N}(u)\right] P_{N} \mathscr{G}_{\alpha}^{N}(u)\right), \quad \mathscr{G}_{0}^{N}(u)=u .
$$

By convention $P_{\infty}=$ Id. It is immediate to show that, for all $N \in \mathbb{N}$ the flow map is globally (in time) well defined, since the frequencies $>N$ evolves under the identity map and the frequencies $\leqslant N$ evolves as the solution of an ODE with conserved $L^{2}$ norm (see Lemma 2.1). In the case $N=\infty$ this is a consequence of the explicit representation formula (1.1). An immediate consequence of (2.2) is that

$$
\left\|\mathscr{G}_{\alpha}^{N} u\right\|_{L^{2}}=\|u\|_{L^{2}}, \quad \forall \alpha \in \mathbb{R},
$$

note, however, that $|u| \neq\left|\mathscr{G}_{\alpha}^{N} u\right|$, which is only the case when $N=\infty$ (see again (1.1)).

It is also clear, looking at the definition (2.1), that the map $\alpha \rightarrow \mathscr{G}_{\alpha}^{N}$ is a one parameter group of transformations, for all $N \in \mathbb{N} \cup\{\infty\}$.

Lemma 2.1. Let $N \in \mathbb{N} \cup\{\infty\}$. For all $u \in L^{2}(\mathbb{T})$ we have

$$
\left\|P_{N} \mathscr{G}_{\alpha}^{N} u\right\|_{L^{2}}=\left\|P_{N} u\right\|_{L^{2}}
$$

For the proof of (2.2) we refer to [12, Section 6]. Moreover, we have the following $L^{2}$-stability result.

Lemma 2.2. Let $N \in \mathbb{N} \cup\{\infty\}$. Then

$$
\left\|\mathscr{G}_{\alpha}^{N} u-\mathscr{G}_{\alpha}^{N} v\right\|_{L^{2}} \lesssim e^{C|\alpha|\left(\left\|P_{N} u\right\|_{L^{2}}^{2}+\left\|P_{N} v\right\|_{L^{2}}^{2}\right)}\|(u-v)\|_{L^{2}}
$$

Proof. Until the end of the proof $N \in \mathbb{N} \cup\{\infty\}$. Decomposing

$$
\begin{aligned}
\mathscr{G}_{\alpha}^{N} u-\mathscr{G}_{\alpha}^{N} v & =P_{N} \mathscr{G}_{\alpha}^{N} u-P_{N} \mathscr{G}_{\alpha}^{N} v+\left(\operatorname{Id}-P_{N}\right)\left(\mathscr{G}_{\alpha}^{N} u\right)-\left(\operatorname{Id}-P_{N}\right)\left(\mathscr{G}_{\alpha}^{N} v\right) \\
& =P_{N} \mathscr{G}_{\alpha}^{N} u-P_{N} \mathscr{G}_{\alpha}^{N} v+\left(\operatorname{Id}-P_{N}\right)(u-v),
\end{aligned}
$$

where the second identity follows by the fact that $\mathscr{G}_{\alpha}^{N}$ is the identity on the frequencies $>N$ (remember definition (2.1)), the (2.3) follows by

$$
\left\|P_{N} \mathscr{G}_{\alpha}^{N} u-P_{N} \mathscr{G}_{\alpha}^{N} v\right\|_{L^{2}} \lesssim e^{C|\alpha|\left(\left\|P_{N} u\right\|_{L^{2}}^{2}+\left\|P_{N} v\right\|_{L^{2}}^{2}\right)}\left\|P_{N}(u-v)\right\|_{L^{2}} .
$$

To prove (2.4) we will need the inequalities

$$
\left\|\mathcal{I}\left[P_{N} \mathscr{G}_{\alpha}^{N} u\right]\right\|_{L^{\infty}} \lesssim\left\|P_{N} \mathscr{G}_{\alpha}^{N} u\right\|_{L^{2}}^{2}=\left\|P_{N} u\right\|_{L^{2}}^{2},
$$

and

$$
\begin{aligned}
\left\|\mathcal{I}\left[P_{N} \mathscr{G}_{\alpha}^{N} u\right]-\mathcal{I}\left[P_{N} \mathscr{G}_{\alpha}^{N} v\right]\right\|_{L^{\infty}} & \lesssim\left\|P_{N} \mathscr{G}_{\alpha}^{N} u+P_{N} \mathscr{G}_{\alpha}^{N} v\right\|_{L^{2}}\left\|P_{N} \mathscr{G}_{\alpha}^{N} u-P_{N} \mathscr{G}_{\alpha}^{N} v\right\|_{L^{2}} \\
& \lesssim\left(\left\|P_{N} u\right\|_{L^{2}}+\left\|P_{N} v\right\|_{L^{2}}\right)\left\|P_{N} \mathscr{G}_{\alpha}^{N} u-P_{N} \mathscr{G}_{\alpha}^{N} v\right\|_{L^{2}} .
\end{aligned}
$$


These follow immediately recalling the form (1.2) of $\mathcal{I}[\cdot]$ and (2.2). Let

$$
\delta_{\alpha}^{N}(u, v):=P_{N} \mathscr{G}_{\alpha}^{N} u-P_{N} \mathscr{G}_{\alpha}^{N} v .
$$

Notice that $\delta_{\alpha}^{N}(u, v)$ solves

$$
\frac{d}{d \alpha} \delta_{\alpha}^{N}(u, v)=i P_{N}\left(\mathcal{I}\left[P_{N} \mathscr{G}_{\alpha}^{N} u\right] \delta_{\alpha}^{N}(u, v)+\left(\mathcal{I}\left[P_{N} \mathscr{G}_{\alpha}^{N} u\right]-\mathcal{I}\left[P_{N} \mathscr{G}_{\alpha}^{N} v\right]\right) P_{N} \mathscr{G}_{\alpha}^{N} v\right)
$$

Pairing this in $L^{2}$ with $\delta_{\alpha}^{N}(u, v)$ we get

$\frac{d}{d \alpha}\left\|\delta_{\alpha}^{N}(u, v)\right\|_{L^{2}}^{2}=2 \operatorname{Re} i\left(\int \mathcal{I}\left[P_{N} \mathscr{G}_{\alpha}^{N} u\right]\left|\delta_{\alpha}^{N}(u, v)\right|^{2}+\int\left(\mathcal{I}\left[P_{N} \mathscr{G}_{\alpha}^{N} u\right]-\mathcal{I}\left[P_{N} \mathscr{G}_{\alpha}^{N} v\right]\right)\left(P_{N} \mathscr{G}_{\alpha}^{N} v\right) \overline{\delta_{\alpha}^{N}(u, v)}\right)$.

Using the Hölder and Cauchy-Schwartz inequalities and (2.5), (2.6), we arrive to

$$
\begin{aligned}
\frac{d}{d \alpha}\left\|\delta_{\alpha}^{N}(u, v)\right\|_{L^{2}}^{2} & \leqslant\left\|\mathcal{I}\left[P_{N} \mathscr{G}_{\alpha}^{N} u\right]\right\|_{L^{\infty}}\left\|\delta_{\alpha}^{N}(u, v)\right\|_{L^{2}}^{2} \\
& +\left\|\mathcal{I}\left[P_{N} \mathscr{G}_{\alpha}^{N} u\right]-\mathcal{I}\left[P_{N} \mathscr{G}_{\alpha}^{N} v\right]\right\|_{L^{\infty}}\left\|P_{N^{\prime}} \mathscr{G}_{\alpha}^{N} v\right\|_{L^{2}}\left\|\delta_{\alpha}^{N}(u, v)\right\|_{L^{2}} \\
& \lesssim\left(\left\|P_{N} u\right\|_{L^{2}}^{2}+\left\|P_{N} v\right\|_{L^{2}}^{2}\right)\left\|\delta_{\alpha}^{N}(u, v)\right\|_{L^{2}}^{2}
\end{aligned}
$$

so that (2.4) follows by Grönwall's lemma.

The flow $\mathscr{G}_{\alpha}^{N}$ approximates $\mathscr{G}_{\alpha}^{\infty}=\mathscr{G}_{\alpha}$ for large $N$ in the $L^{2}(\mathbb{T})$ topology, uniformly on compact sets. This is proved in Lemma 2.4. Before we need the following statement.

Lemma 2.3. The map

$$
(\alpha, u) \in \mathbb{R} \times L^{2}(\mathbb{T}) \rightarrow \mathscr{G}_{\alpha} u \in L^{2}(\mathbb{T})
$$

is continuous.

Proof. Decomposing

$$
\left\|\mathscr{G}_{\alpha} u-\mathscr{G}_{\beta} v\right\| \leqslant\left\|\mathscr{G}_{\alpha} u-\mathscr{G}_{\alpha} v\right\|+\left\|\mathscr{G}_{\alpha} v-\mathscr{G}_{\beta} v\right\|
$$

The statement easily follows by the estimate (2.4) and

$$
\left\|\mathscr{G}_{\alpha} v-\mathscr{G}_{\beta} v\right\|_{L^{2}} \lesssim|\alpha-\beta|\|v\|_{L^{2}}^{3} .
$$

To prove this we assume $\beta<\alpha$ and we integrate (2.1) over $[\beta, \alpha]$, so that

$$
\mathscr{G}_{\alpha} v-\mathscr{G}_{\beta} v=i \int_{\beta}^{\alpha} \mathcal{I}\left[\mathscr{G}_{\alpha^{\prime}}(v)\right] \mathscr{G}_{\alpha^{\prime}}(v) d \alpha^{\prime} .
$$

Taking the $L^{2}$ norm of this identity and using Minkowsky's and Hölder inequalities and (2.5), (2.2) we arrive to

$$
\begin{aligned}
\left\|\mathscr{G}_{\alpha} v-\mathscr{G}_{\beta} v\right\|_{L^{2}} & \leqslant \int_{\beta}^{\alpha}\left\|\left(\mathcal{I}\left[\mathscr{G}_{\alpha^{\prime}}(v)\right] \mathscr{G}_{\alpha^{\prime}}(v)\right)\right\|_{L^{2}} d \alpha^{\prime} \\
& \leqslant \int_{\beta}^{\alpha}\left\|\mathcal{I}\left[\mathscr{G}_{\alpha^{\prime}}(v)\right]\right\|_{L^{\infty}}\left\|\mathscr{G}_{\alpha^{\prime}}(v)\right\|_{L^{2}} d \alpha^{\prime} \leqslant \int_{\beta}^{\alpha}\|v\|_{L^{2}}^{3} d \alpha^{\prime}=|\alpha-\beta|\|v\|_{L^{2}}^{3},
\end{aligned}
$$

as claimed.

Lemma 2.4. Let $N \in \mathbb{N}$ and $\bar{\alpha} \geqslant 0$. Let $A$ be a compact subset of $L^{2}(\mathbb{T})$. Then

$$
\lim _{N \rightarrow \infty} \sup _{u \in A,|\alpha| \leqslant \bar{\alpha}}\left\|\mathscr{G}_{\alpha} u-\mathscr{G}_{\alpha}^{N} u\right\|_{L^{2}}=0 .
$$


Proof. We decompose

$$
\begin{aligned}
\mathscr{G}_{\alpha} u-\mathscr{G}_{\alpha}^{N} u & =P_{N} \mathscr{G}_{\alpha} u-P_{N} \mathscr{G}_{\alpha}^{N} u+\left(\operatorname{Id}-P_{N}\right)\left(\mathscr{G}_{\alpha} u-\mathscr{G}_{\alpha}^{N} u\right) \\
& =P_{N} \mathscr{G}_{\alpha} u-P_{N} \mathscr{G}_{\alpha}^{N} u+\left(\operatorname{Id}-P_{N}\right)\left(\mathscr{G}_{\alpha} u-u\right),
\end{aligned}
$$

where the second identity follows by the fact that $\mathscr{G}_{\alpha}^{N}$ is the identity on the frequencies $>N$ (remember definition (2.1)).

Since $A$ is compact, it is in particular bounded, so that $\|u\|_{L^{2}} \leqslant R$ for some $R>1$, and by (2.2) also $\left\|\mathscr{G}_{\alpha} u\right\|_{L^{2}} \leqslant R$. Thus we have

$$
\lim _{N \rightarrow \infty} \sup _{u \in A,|\alpha| \leqslant \bar{\alpha}}\left\|\left(\operatorname{Id}-P_{N}\right) \mathscr{G}_{\alpha} u\right\|_{L^{2}}+\left\|\left(\operatorname{Id}-P_{N}\right) u\right\|_{L^{2}}=0
$$

so that the (2.7) follows by

$$
\lim _{N \rightarrow \infty} \sup _{u \in A,|\alpha| \leqslant \bar{\alpha}}\left\|P_{N} \mathscr{G}_{\alpha} u-P_{N} \mathscr{G}_{\alpha}^{N} u\right\|_{L^{2}}=0 .
$$

To prove (2.9) we will need the inequalities (2.5) and

$$
\left\|\mathcal{I}\left[\mathscr{G}_{\alpha} u\right]-\mathcal{I}\left[P_{N} \mathscr{G}_{\alpha}^{N} u\right]\right\|_{L^{\infty}}^{2} \lesssim R^{2}\left\|P_{N} \mathscr{G}_{\alpha} u-P_{N} \mathscr{G}_{\alpha}^{N} u\right\|_{L^{2}}^{2}+R^{2}\left\|\left(\operatorname{Id}-P_{N}\right) \mathscr{G}_{\alpha} u\right\|_{L^{2}}^{2},
$$

valid for $N \in \mathbb{N} \cup\{\infty\}$, which follows by

$$
\begin{aligned}
\left\|\mathcal{I}\left[\mathscr{G}_{\alpha} u\right]-\mathcal{I}\left[P_{N} \mathscr{G}_{\alpha}^{N} u\right]\right\|_{L^{\infty}} & \lesssim\left\|\mathscr{G}_{\alpha} u+P_{N} \mathscr{G}_{\alpha}^{N} u\right\|_{L^{2}}\left\|\mathscr{G}_{\alpha} u-P_{N} \mathscr{G}_{\alpha}^{N} u\right\|_{L^{2}} \\
& \lesssim R\left\|\mathscr{G}_{\alpha} u-P_{N} \mathscr{G}_{\alpha}^{N} u\right\|_{L^{2}},
\end{aligned}
$$

which easily follows by the definition (1.2) of $\mathcal{I}[\cdot]$ and (2.2). Let

$$
\delta_{\alpha}^{N} u:=P_{N} \mathscr{G}_{\alpha} u-P_{N} \mathscr{G}_{\alpha}^{N} u \text {. }
$$

Notice that $\delta_{\alpha}^{N} u$ solves

$$
\frac{d}{d \alpha} \delta_{\alpha}^{N} u=i P_{N}\left(\mathcal{I}\left[\mathscr{G}_{\alpha} u\right] \delta_{\alpha}^{N} u+\left(\mathcal{I}\left[\mathscr{G}_{\alpha} u\right]-\mathcal{I}\left[P_{N} \mathscr{G}_{\alpha}^{N} u\right]\right) P_{N} \mathscr{G}_{\alpha}^{N} u\right) .
$$

Pairing this in $L^{2}$ with $\delta_{\alpha}^{N} u$ we get

$$
\frac{d}{d \alpha}\left\|\delta_{\alpha}^{N} u\right\|_{L^{2}}^{2}=2 \operatorname{Re} i\left(\int \mathcal{I}\left[\mathscr{G}_{\alpha} u\right]\left|\delta_{\alpha}^{N} u\right|^{2}+\int\left(\mathcal{I}\left[\mathscr{G}_{\alpha} u\right]-\mathcal{I}\left[P_{N} \mathscr{G}_{\alpha}^{N} u\right]\right) P_{N}\left(\mathscr{G}_{\alpha}^{N} u\right) \overline{\delta_{\alpha}^{N} u}\right) .
$$

Using the Hölder and Cauchy-Schwartz inequalities and (2.5), (2.10), we arrive to

$$
\begin{aligned}
\frac{d}{d \alpha}\left\|\delta_{\alpha}^{N} u\right\|_{L^{2}}^{2} & \lesssim\left\|\mathcal{I}\left[\mathscr{G}_{\alpha} u\right]\right\|_{L^{\infty}}\left\|\delta_{\alpha}^{N} u\right\|_{L^{2}}^{2}+\left\|\mathcal{I}\left[\mathscr{G}_{\alpha} u\right]-\mathcal{I}\left[P_{N} \mathscr{G}_{\alpha}^{N} u\right]\right\|_{L^{\infty}}\left\|P_{N} \mathscr{G}_{\alpha}^{N} u\right\|_{L^{2}}\left\|\delta_{\alpha}^{N} u\right\|_{L^{2}} \\
& \left.\lesssim R^{2} \|\left(\operatorname{Id}-P_{N}\right) \mathscr{G}_{\alpha} u\right)\left\|_{L^{2}}^{2}+R^{2}\right\| \delta_{\alpha}^{N} u \|_{L^{2}}^{2} .
\end{aligned}
$$

Thus, using the fact that $\left.\delta_{\alpha}^{N} u\right|_{\alpha=0}=0$, the Grönwall's inequality gives

$$
\left.\left\|\delta_{\alpha}^{N} u\right\|_{L^{2}}^{2} \lesssim R^{2} e^{R^{2}|\bar{\alpha}|} \int_{0}^{\alpha} \|\left(\operatorname{Id}-P_{N}\right) \mathscr{G}_{\alpha^{\prime}} u\right) \|_{L^{2}}^{2} d \alpha^{\prime}, \quad|\alpha| \leqslant|\bar{\alpha}| .
$$

Recalling (2.8), (2.2) and using dominated convergence, it is clear that the right hand side of (2.12) goes to zero as $N \rightarrow \infty$. On the other hand, using Lemma 2.3, it is clear that the maps

$$
\left.(\alpha, u) \in[-\bar{\alpha}, \bar{\alpha}] \times A \rightarrow \Xi^{N}(\alpha, u):=\int_{0}^{\alpha} R^{2} \|\left(\operatorname{Id}-P_{N}\right) \mathscr{G}_{\alpha^{\prime}} u\right) \|_{L^{2}}^{2} d \alpha^{\prime}
$$

are continuous, for all $N \in \mathbb{N}$. Since $\Xi^{N}$ are defined on a compact set, are monotonic (w.r.t. $N$ ) and they vanish in the limit $N \rightarrow \infty$ (as we have just proved), by Dini's theorem we have that they converge to zero uniformly. Recalling (2.12), this complete the proof.

The next result is a direct corollary of Lemma 2.4. 
Corollary 2.5. Let $\varepsilon>0$ and $\bar{\alpha} \geqslant 0$. For all compact subset $A$ of $L^{2}(\mathbb{T})$, there exists $N^{*}$ such that

$$
\mathscr{G}_{\alpha}(A) \subset \mathscr{G}_{\alpha}^{N}(A+B(\varepsilon)) .
$$

for all $|\alpha| \leqslant \bar{\alpha}$ and for all $N>N^{*}$.

Proof. Let $u \in A$ and $u^{N}:=\mathscr{G}_{-\alpha}^{N} \mathscr{G}_{\alpha} u$. Since

$$
\mathscr{G}_{\alpha}^{N} u^{N}=\mathscr{G}_{\alpha} u
$$

it suffices to prove

$$
\left\|u-u^{N}\right\|_{L^{2}} \leqslant \varepsilon
$$

for all sufficiently large $N$, uniformly in $u \in A,|\alpha| \leqslant \bar{\alpha}$. Indeed, if (2.14) holds, it means that for all $u \in A$ we have found

$$
u^{N} \in u+B(\varepsilon) \subseteq A+B(\varepsilon)
$$

such that (see (2.13))

$$
\mathscr{G}_{\alpha} u=\mathscr{G}_{\alpha}^{N} u^{N} \in \mathscr{G}_{\alpha}^{N}(A+B(\varepsilon)) .
$$

To prove (2.14) we notice that, since $A$ is a compact subset of $L^{2}$ we have $\|u\|_{L^{2}} \leqslant C_{A}$ for all $u \in A$. Thus

$$
\begin{aligned}
\lim _{N} \sup _{u \in A,|\alpha| \leqslant \bar{\alpha}}\left\|u-u^{N}\right\|_{L^{2}} & \left.=\lim _{N} \sup _{u \in A,|\alpha| \leqslant \bar{\alpha}} \| \mathscr{G}_{-\alpha}^{N} \mathscr{G}_{\alpha}^{N} u-\mathscr{G}_{-\alpha}^{N} \mathscr{G}_{\alpha} u\right) \|_{L^{2}} \\
& \lesssim \lim _{N} \sup _{u \in A} e^{C \bar{\alpha}\left(\left\|\mathscr{G}_{\alpha}^{N} u\right\|_{L^{2}}+\left\|P_{N} \mathscr{G}_{\alpha} u\right\|_{L^{2}}\right)}\left\|\mathscr{G}_{\alpha}^{N} u-\mathscr{G}_{\alpha} u\right\|_{L^{2}} \\
& \leqslant e^{2 C C_{A}^{2} \bar{\alpha}} \lim _{N} \sup _{u \in A}\left\|\mathscr{G}_{\alpha}^{N} u-\mathscr{G}_{\alpha} u\right\|_{L^{2}}=0 .
\end{aligned}
$$

where we used (2.3) in the first inequality, (2.2) in the second inequality and (2.7) to take the limit over $N$. This completes the proof of Corollary 2.5.

\section{Proof of Theorem 1.1}

Here we give the main argument to prove Theorem 1.1, leaving all the (many) auxiliary statements to the next sections. We follow the strategy introduced in [33].

First we define the measure

$$
\tilde{\gamma}_{s, N}(A):=E_{s}\left[1_{\left\{A \cap\left\{\left\|P_{N} u\right\|_{L^{2}} \leqslant R\right\}\right.}\right], \quad A \in \mathscr{B}\left(L^{2}(\mathbb{T})\right) .
$$

Recall that $\tilde{\gamma}_{s, N}(A)$ also depends on $R$, even though we will not track this dependence to simplify the notations.

Using the group property of $\left\{\mathscr{G}_{\alpha}^{N}\right\}_{\alpha \in \mathbb{R}}$ we can easily check that

$$
\left.\frac{d}{d \alpha}\left(\tilde{\gamma}_{s, N} \circ \mathscr{G}_{\alpha}^{N}\right)(A)\right|_{\alpha=\bar{\alpha}}=\left.\frac{d}{d \alpha}\left(\tilde{\gamma}_{s, N} \circ \mathscr{G}_{\alpha}^{N}\right)\left(\mathscr{G}_{\bar{\alpha}}^{N} A\right)\right|_{\alpha=0} .
$$

Now we use the factorisation (1.13) and Proposition 4.1 of [33], so that for all $E \subset \mathscr{B}\left(L^{2}\right)$ we have

$$
\begin{aligned}
& \tilde{\gamma}_{s, N}\left(\mathscr{G}_{\alpha}^{N}(E)\right)=\int_{\mathscr{G}_{\alpha}^{N}(E)} \gamma_{s}(d u) 1_{\left\{\left\|P_{N} u\right\|_{L^{2}} \leqslant R \|\right\}} \\
& =\int_{E} L_{N}\left(d P_{N} u\right) \gamma_{s}^{\perp, N}\left(d P_{>N} u\right) 1_{\left\{\left\|P_{N} u\right\|_{L^{2}} \leqslant R \|\right\}}\left|\operatorname{det} D \mathscr{G}_{\alpha}^{N}(u)\right| \exp \left(-\frac{1}{2}\left\|P_{N^{\prime}} \mathscr{G}_{\alpha}^{N} u\right\|_{\dot{H}_{s}}^{2}-\frac{1}{2}\left\|P_{N^{\prime}} u\right\|_{L^{2}}^{2}\right), \\
& =\int_{E} \tilde{\gamma}_{s, N}(d u)\left|\operatorname{det} D P_{N} \mathscr{G}_{\alpha}^{N}(u)\right| \exp \left(\frac{1}{2}\left\|P_{N} u\right\|_{\dot{H}^{s}}^{2}-\frac{1}{2}\left\|P_{N} \mathscr{G}_{\alpha}^{N} u\right\|_{\dot{H}^{s}}^{2}\right)
\end{aligned}
$$


where $D P_{N} \mathscr{G}_{\alpha}^{N}(u)$ denotes the Jacobian matrix associated to $P_{N} \mathscr{G}_{\alpha}^{N}$ and in the second identity we used (2.2). Using this identity with $E=\mathscr{G}_{\bar{\alpha}}^{N} A$, we arrive to

$$
\tilde{\gamma}_{s, N} \circ \mathscr{G}_{\alpha}^{N}\left(\mathscr{G}_{\bar{\alpha}}^{N} A\right)=\int_{\mathscr{G}_{\bar{\alpha}}^{N} A}\left|\operatorname{det} D P_{N} \mathscr{G}_{\alpha}^{N}(u)\right| \exp \left(\frac{1}{2}\left\|P_{N} u\right\|_{\dot{H}^{s}}^{2}-\frac{1}{2}\left\|P_{N} \mathscr{G}_{\alpha}^{N} u\right\|_{\dot{H}^{s}}^{2}\right) \tilde{\gamma}_{s, N}(d u)
$$

so that, using (4.2) to compute the derivative in $d \alpha$ at $\alpha=0$ of $\left|\operatorname{det} D P_{N} \mathscr{G}_{\alpha}^{N}(u)\right|$, we can rewrite $(3.2)$ as

$$
\begin{aligned}
& \left.\frac{d}{d \alpha}\left(\tilde{\gamma}_{s, N} \circ \mathscr{G}_{\alpha}^{N}\right)\left(\mathscr{G}_{\bar{\alpha}}^{N} A\right)\right|_{\alpha=0} \\
& \left.=\int_{\mathscr{G}_{\bar{\alpha}}^{N} A} \tilde{\gamma}_{s, N}(d u) \eta(u) \operatorname{div} P_{N}\left(\mathcal{I}\left[P_{N} u\right] P_{N} u\right)\right)+\left.\int_{\mathscr{G}_{\bar{\alpha}}^{N} A} \tilde{\gamma}_{s, N}(d u) \frac{d}{d \alpha}\left\|P_{N} \mathscr{G}_{\alpha}^{N} u\right\|_{\dot{H}^{s}}^{2}\right|_{\alpha=0} \\
& \left.=\int_{\mathscr{G}_{\bar{\alpha}}^{N} A} \tilde{\gamma}_{s, N}(d u) \eta(u) \operatorname{div} P_{N}\left(\mathcal{I}\left[P_{N} u\right] P_{N} u\right)\right)+\left.\int_{\mathscr{G}_{\bar{\alpha}}^{N} A} \tilde{\gamma}_{s, N}(d u) \frac{d}{d \alpha}\left\|\mathscr{G}_{\alpha} P_{N} u\right\|_{\dot{H}^{s}}^{2}\right|_{\alpha=0}
\end{aligned}
$$

where $\eta(u):=i \frac{\operatorname{det} D P_{N} \mathscr{G}_{\alpha}^{N}(u)}{\left|\operatorname{det} D P_{N} \mathscr{G}_{\alpha}^{N}(u)\right|}$, so in particular $|\eta|=1$, and we also used

$$
\left.\frac{d}{d \alpha}\left\|P_{N} \mathscr{G}_{\alpha}^{N} u\right\|_{\dot{H}^{s}}^{2}\right|_{\alpha=0}=\left.\frac{d}{d \alpha}\left\|\mathscr{G}_{\alpha} P_{N} u\right\|_{\dot{H}^{s}}^{2}\right|_{\alpha=0} .
$$

To prove (3.4), bearing in mind (1.8), (1.10), (2.1), we observe that

$$
\begin{aligned}
& \left.\frac{d}{d \alpha}\left\|P_{N} \mathscr{G}_{\alpha}^{N} u\right\|_{\dot{H}^{s}}^{2}\right|_{\alpha=0}=\left.2 \operatorname{Re} \int\left(\overline{P_{N} \mathscr{G}_{\alpha}^{N} u}\right)^{(s)}\left(\frac{d}{d \alpha} P_{N} \mathscr{G}_{\alpha}^{N} u\right)^{(s)}\right|_{\alpha=0} \\
& =\left.2 \operatorname{Re} \int{\overline{P_{N} \mathscr{G}_{\alpha}^{N} u}}^{(s)}\left(i \mathcal{I}\left[P_{N} \mathscr{G}_{\alpha}^{N} u\right] P_{N} \mathscr{G}_{\alpha}^{N} u\right)^{(s)}\right|_{\alpha=0} \\
& =2 \operatorname{Re} \int{\overline{P_{N} u}}^{(s)}\left(i \mathcal{I}\left[P_{N} u\right] P_{N} u\right)^{(s)}= \\
& =\left.2 \operatorname{Re} \int \overline{\mathscr{G}}_{\alpha} P_{N} u{ }^{(s)}\left(i \mathcal{I}\left[\mathscr{G}_{\alpha} P_{N} u\right] \mathscr{G}_{\alpha} P_{N} u\right)^{(s)}\right|_{\alpha=0} \\
& =\left.2 \operatorname{Re} \int\left(\overline{\mathscr{G}_{\alpha} P_{N} u}\right)^{(s)}\left(\frac{d}{d \alpha} \mathscr{G}_{\alpha} P_{N} u\right)^{(s)}\right|_{\alpha=0}=\left.\frac{d}{d \alpha}\left\|\mathscr{G}_{\alpha} P_{N} u\right\|_{\dot{H}^{s}}^{2}\right|_{\alpha=0} .
\end{aligned}
$$

Now, the first summand in (3.3) gives a vanishing contribution as $N \rightarrow \infty$. Indeed by Proposition 4.4 below and Hölder inequality there is $\varepsilon>0$ such that

$$
\int_{\mathscr{G}_{\bar{\alpha}}^{N} A} \tilde{\gamma}_{s, N}(d u)\left|\operatorname{div} P_{N}\left(\mathcal{I}\left[P_{N} u\right] P_{N} u \mid\right)\right| \lesssim \frac{\tilde{\gamma}_{s, N}\left(\mathscr{G}_{\bar{\alpha}}^{N} A\right)^{1-\frac{1}{p}} p}{N^{\varepsilon}}
$$

On the second summand use again Hölder inequality:

$$
\left.\int_{\mathscr{G}_{\bar{\alpha}}^{N} A} \tilde{\gamma}_{s, N}(d u) \frac{d}{d \alpha}\left\|\mathscr{G}_{\alpha} P_{N} u\right\|_{\dot{H}^{s}}^{2}\right|_{\alpha=0} \leqslant \tilde{\gamma}_{s, N}\left(\mathscr{G}_{\bar{\alpha}}^{N} A\right)^{1-\frac{1}{p}}\left\|\frac{d}{d \alpha}\right\| \mathscr{G}_{\alpha} P_{N} u\left\|\left._{\dot{H}^{s}}^{2}\right|_{\alpha=0}\right\| \|_{L^{p}\left(\tilde{\gamma}_{s, N}\right)} .
$$

Hereafter we set

$$
R^{*}:=\max \left(R^{\frac{2}{2 s-1}}, R^{2(2 s-1)}\right) .
$$

Proposition 3.1. Let $s>\frac{1}{2}$ and $R>0$. For all $N$ there is a $C>0$ such that

$$
\left\|\frac{d}{d \alpha}\right\| \mathscr{G}_{\alpha} P_{N} u\left\|\left._{\dot{H}^{s}}^{2}\right|_{\alpha=0}\right\|_{L^{p}\left(\tilde{\gamma}_{s, N}\right)} \leqslant C R^{*} p .
$$

Altogether

$$
\left.\frac{d}{d \alpha}\left(\tilde{\gamma}_{s, N} \circ \mathscr{G}_{\alpha}^{N}\right)(A)\right|_{\alpha=\bar{\alpha}} \leqslant C R^{*} p \tilde{\gamma}_{s, N}\left(\mathscr{G}_{\bar{\alpha}}^{N} A\right)^{1-\frac{1}{p}}
$$


which implies

$$
\left.\frac{d}{d \alpha}\left(\tilde{\gamma}_{s, N} \circ \mathscr{G}_{\alpha}^{N}\right)(A)\right)^{\frac{1}{p}} \leqslant C R^{*} .
$$

Thus

$$
\left(\tilde{\gamma}_{s, N} \circ \mathscr{G}_{\alpha}^{N}\right)(A) \leqslant\left(C R^{*}|\alpha|+\tilde{\gamma}_{s, N}(A)^{\frac{1}{p}}\right)^{p} \leqslant\left(\left(C R^{*}\right)^{p}|\alpha|^{p}+\tilde{\gamma}_{s, N}(A)\right) 2^{p-1} .
$$

Let $\delta>0$ and $\tilde{\gamma}_{s}(A) \leqslant \delta$. Since

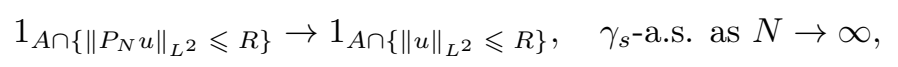

by dominated convergence we have

$$
\left(\tilde{\gamma}_{s, N} \circ \mathscr{G}_{\alpha}^{N}\right)(A) \leqslant\left(\left(C R^{*}\right)^{p}|\alpha|^{p}+2 \delta\right) 2^{p-1},
$$

for all $N$ sufficiently large (the choice of $N$ only depends on $A$ ). Now letting $\bar{\alpha}:=\frac{1}{4 C R^{*}}$ we have that for all $|\alpha| \leqslant \bar{\alpha}$ :

$$
\left(\tilde{\gamma}_{s, N} \circ \mathscr{G}_{\alpha}^{N}\right)(A) \leqslant \frac{1}{2}\left(2^{-p}+\delta 2^{p+1}\right), \quad \forall p>1 .
$$

Therefore for any $\varepsilon \in(0,1 / 2)$ we can take $p=-\log _{2} \varepsilon$ and see that there is $0<\delta<\varepsilon^{2}$ such that

$$
\tilde{\gamma}_{s}(A) \leqslant \delta \quad \Rightarrow \quad\left(\tilde{\gamma}_{s, N} \circ \mathscr{G}_{\alpha}^{N}\right)(A) \leqslant \varepsilon, \quad|\alpha| \leqslant \bar{\alpha} .
$$

To upgrade (3.15) to the limiting version for $N \rightarrow \infty$ we use Corollary 2.5.

Let us take $R>0$ and any compact $A \subset B(R)$, such that $\tilde{\gamma}_{s}(A) \leqslant \delta / 2$. Since $A$ is compact, we can choose a small enough $\varepsilon^{\prime}>0$ such that

$$
\tilde{\gamma}_{s}\left(A+B\left(\varepsilon^{\prime}\right)\right) \leqslant \delta .
$$

By (3.15) for $|\alpha| \leqslant \bar{\alpha}$ we get

$$
\tilde{\gamma}_{s, N}\left(\mathscr{G}_{\alpha}^{N}\left(A+B\left(\varepsilon^{\prime}\right)\right)\right) \leqslant \varepsilon .
$$

Corollary 2.5 and the obvious inclusion $B(R) \subseteq\left\{\left\|P_{N} u\right\|_{2} \leqslant R\right\}$ implies that, for all $N$ sufficiently large (again the choice of $N$ only depends on $A$ ):

$$
\mathscr{G}_{\alpha}(A) \cap B(R) \subset \mathscr{G}_{\alpha}^{N}\left(A+B\left(\varepsilon^{\prime}\right)\right) \cap\left\{\left\|P_{N} u\right\|_{2} \leqslant R\right\} .
$$

Thus

$$
\begin{aligned}
\left(\tilde{\gamma}_{s} \circ \mathscr{G}_{\alpha}\right)(A) & =\gamma_{s}\left(\mathscr{G}_{\alpha}(A) \cap B(R)\right) \\
& \leqslant \gamma_{s}\left(\mathscr{G}_{\alpha}^{N}\left(A+B\left(\varepsilon^{\prime}\right)\right) \cap\left\{\left\|P_{N} u\right\|_{2} \leqslant R\right\}\right)=\tilde{\gamma}_{s, N}\left(\mathscr{G}_{\alpha}^{N}\left(A+B\left(\varepsilon^{\prime}\right)\right)\right) \leqslant \varepsilon .
\end{aligned}
$$

In conclusion there exists $\bar{\alpha}$ such that the following holds. For all $\varepsilon \in(0,1 / 2)$ we can take $0<\delta<\varepsilon^{2}$ such that for any $|\alpha|<|\bar{\alpha}|$ and for any compact $A \subset B(R)$, we have

$$
\tilde{\gamma}_{s}(A) \leqslant \delta \quad \Rightarrow \quad\left(\tilde{\gamma}_{s} \circ \mathscr{G}_{\alpha}\right)(A) \leqslant \varepsilon .
$$

We can extend the previous relation to any $A \in \mathscr{B}\left(L^{2}(\mathbb{T})\right) \cap B(R)$ using the regularity of $\tilde{\gamma}_{s}$ (inherited by $\gamma_{s}$ ) by the general procedure explained in [33, Lemma 8.1], which easily adapts here. This proves the local almost invariance of $\tilde{\gamma}_{s}$ under $\mathscr{G}_{\alpha},|\alpha| \leqslant \bar{\alpha}$. Since $\bar{\alpha}$ only depends on $R$ and the restriction $u \in B(R)$ is invariant under $\mathscr{G}_{\alpha}$, we can globalise to $\alpha \in \mathbb{R}$ by the usual gluing procedure.

Therefore we have shown (1.7), where the density $\rho_{\alpha}$ is in $L^{1}\left(\tilde{\gamma}_{s}\right)$. It remains to prove there exists $p_{0}>1$ such that the density lies in all the spaces $L^{p}\left(\tilde{\gamma}_{s}\right)$ for $p \in\left[1, p_{0}\right)$. First of all we start by a somewhat more quantitative version of (3.15).

Lemma 3.2. Let $s>1 / 2$. There exist $\alpha_{0}>0$ such that the following holds. For all $|\alpha|<\alpha_{0}$ and for all $A \in \mathscr{B}\left(L^{2}\right) \cap B(R)$ one has

$$
\left(\tilde{\gamma}_{s} \circ \mathscr{G}_{\alpha}\right)(A) \leqslant 2 \tilde{\gamma}_{s}(A)^{1 / 2} .
$$

More precisely we can choose $\alpha_{0}=c / R^{*}$, where $c>0$ is an absolute constant and $R^{*}$ is a function of the mass $R$ defined in (3.8). 
Proof. Let fix $A$ and let us start again by (see (3.12))

$$
\left(\tilde{\gamma}_{s, N} \circ \mathscr{G}_{\alpha}^{N}\right)(A) \leqslant\left(C R^{*}|\alpha|+\tilde{\gamma}_{s, N}(A)^{\frac{1}{p}}\right)^{p} .
$$

We can assume $\tilde{\gamma}_{s}(A)>0$, otherwise (3.18) is consequence of (3.17). Thus, since

$$
\tilde{\gamma}_{s, N}(A) \rightarrow \tilde{\gamma}_{s}(A)>0 \text { as } N \rightarrow \infty
$$

we have, for all sufficiently large $N$ (the choice of $N$ only depends on $A$ )

$$
\tilde{\gamma}_{s, N}(A) \leqslant 2 \tilde{\gamma}_{s}(A)
$$

Thus we can bound the right hand side of (3.19) as

$$
\left(C R^{*}|\alpha|+\left(2 \tilde{\gamma}_{s}(A)\right)^{\frac{1}{p}}\right)^{p}=2 \tilde{\gamma}_{s}(A)\left(1+\frac{2 C R^{*}|\alpha|}{\left(2 \tilde{\gamma}_{s}(A)\right)^{\frac{1}{p}}}\right)^{p}=2 \tilde{\gamma}_{s}(A) e^{p \log \left(1+C R^{*}|\alpha|\left(2 \tilde{\gamma}_{s}(A)\right)^{-\frac{1}{p}}\right)} .
$$

Now we can pick

$$
p=p(A)=\log \frac{1}{2 \tilde{\gamma}_{s}(A)} \quad \text { such that } \quad\left(2 \tilde{\gamma}_{s}(A)\right)^{-\frac{1}{p}}=e
$$

Thus

$$
\left(\tilde{\gamma}_{s, N} \circ \mathscr{G}_{\alpha}^{N}\right)(A) \leqslant 2 \tilde{\gamma}_{s}\left(P_{N} A\right) e^{p \log \left(1+C R^{*} e \alpha\right)} \leqslant 2 \tilde{\gamma}_{s}\left(P_{N} A\right) e^{p C R^{*} e \alpha} .
$$

Then we claim that

$$
e^{p C R^{*} e \alpha} \leqslant \tilde{\gamma}_{s}(A)^{-1 / 2}
$$

To have that, it must be

$$
p C R^{*} e \alpha \leqslant \frac{1}{2} \log \frac{1}{\tilde{\gamma}_{s}(A)}=\frac{p}{2}
$$

which is true for $|\alpha| \leqslant \alpha_{0}$ with $\alpha_{0}=c / R^{*}$ and $c$ sufficiently small. Plugging (3.23) into (3.22) we arrive to

$$
\left(\tilde{\gamma}_{s, N} \circ \mathscr{G}_{\alpha}^{N}\right)(A) \leqslant 2 \tilde{\gamma}_{s}(A)^{1 / 2}, \quad|\alpha| \leqslant \alpha_{0} .
$$

Finally we upgrade (3.25) to (3.18) using Corollary 2.5 as in the non quantitative argument above.

The size of $\alpha_{0}$ in Lemma (3.2) can be arbitrarily increased but paying an arbitrarily small factor loss on the exponent on the right hand side of (3.18) [25, Remark 5.6]. We have the following

Lemma 3.3. Let $s>1 / 2$ and $\alpha \in \mathbb{R}$. There exist an absolute constant $\bar{C}>1$ such that for all $A \in \mathscr{B}\left(L^{2}\right) \cap B(R)$ one has

$$
\left(\tilde{\gamma}_{s} \circ \mathscr{G}_{\alpha}\right)(A) \leqslant 4 \tilde{\gamma}_{s}(A) \frac{1}{\overline{\bar{C}}^{*}|\alpha|}
$$

Proof. We can assume $\alpha \geqslant 0$. Let $\alpha_{0}$ be given as in the previous lemma. We can also assume $\alpha_{0} \in(0,1)$. Let now define $M$ as the largest integer such that $\alpha_{0} M \leqslant \alpha$. We will show that for all $M \in \mathbb{N} \cup\{0\}$ we have

$$
\left(\tilde{\gamma}_{s} \circ \mathscr{G}_{\alpha}\right)(A) \leqslant 2^{\sum_{j=0}^{M} 2^{-j}} \tilde{\gamma}_{s}(A)^{2^{-(M+1)}}, \quad \text { for } \alpha \in\left[\alpha_{0} M, \alpha_{0}(M+1)\right] .
$$

Since $\alpha_{0} M \geqslant \alpha-\alpha_{0}$ (by definition of $M$ ) one has $2^{-(M+1)} \geqslant 2^{-\frac{1}{\alpha_{0}}\left(\alpha+\alpha_{0}\right)}>2^{-\frac{1}{\alpha_{0}}(\alpha+1)}$ which means that the (3.26) follows by (3.27), recalling that $\alpha_{0}=c / R^{*}$ for some absolute small constant $c>0$.

It remains to prove (3.27). When $M=0$ (which means that $0 \leqslant \alpha \leqslant \alpha_{0}$ ), the (3.27) follows by (3.18). Let assume we have proved (3.27) up to $M-1$. In particular we have

$$
\tilde{\gamma}_{s}\left(\mathscr{G}_{\alpha_{0} M}(A)\right)=\left(\tilde{\gamma}_{s} \circ \mathscr{G}_{\alpha_{0} M}\right)(A)=\left(\tilde{\gamma}_{s}\left(\mathscr{G}_{\alpha_{0} M}(A)\right)\right)^{1 / 2} \leqslant 2^{\sum_{j=0}^{M-1} 2^{-j}} \tilde{\gamma}_{s}(A)^{2^{-M}}
$$

Writing $\alpha \in\left[\alpha_{0} M, \alpha_{0}(M+1)\right]$ as $\alpha=\alpha_{0} M+\alpha^{\prime}$ with $\left|\alpha^{\prime}\right| \leqslant \alpha_{0}$ and using (3.18)-(3.28) we have

$$
\begin{aligned}
\left(\tilde{\gamma}_{s} \circ \mathscr{G}_{\alpha}\right)(A) & =\left(\tilde{\gamma}_{s} \circ \mathscr{G}_{\alpha^{\prime}}\right)\left(\mathscr{G}_{\alpha_{0} M}(A)\right) \leqslant 2\left(\tilde{\gamma}_{s}\left(\mathscr{G}_{\alpha_{0} M}(A)\right)\right)^{1 / 2} \\
& \leqslant 2\left(2^{\sum_{j=0}^{M-1} 2^{-j}} \tilde{\gamma}_{s}(A)^{2^{-M}}\right)^{1 / 2} \leqslant 2^{\sum_{j=0}^{M} 2^{-j}} \tilde{\gamma}_{s}(A)^{2^{-(M+1)}}
\end{aligned}
$$


as claimed.

We are now ready to prove that the density $\rho_{\alpha}$ is slightly more than just integrable, for all $\alpha \in \mathbb{R}$.

Proposition 3.4. Let $s>1 / 2, R>0$ and $\alpha \in \mathbb{R}$. There exists $p_{0}(|\alpha|, R)>1$ such that

$$
\rho_{\alpha} \in L^{p}\left(\gamma_{s}\right) \quad \text { for all } p<p_{0}(|\alpha|, R) \text {. }
$$

In fact we have $p_{0}(|\alpha|, R) \rightarrow 1$ as $|\alpha| \rightarrow \infty$.

Proof. With reference to (3.26) we let for brevity

so that it becomes

$$
1-\delta:=\frac{1}{\bar{C}^{R^{*}|\alpha|}}
$$

$\left(\tilde{\gamma}_{s} \circ \mathscr{G}_{\alpha}\right)(A) \lesssim \tilde{\gamma}_{s}(A)^{1-\delta}$.

Since $\bar{C}>1$ we have $\delta \in(0,1)$ and $\delta \rightarrow 1$ as $\alpha \rightarrow \infty$. Let now $\lambda>0$ and set

$$
A_{\lambda, \alpha}:=\left\{u: \rho_{\alpha}(u)>\lambda\right\} .
$$

Therefore using (3.30) we have for $|\alpha|<\alpha_{0}$

$$
\tilde{\gamma}_{s}\left(A_{\lambda, \alpha}\right)=\frac{1}{\lambda} \int_{A_{\lambda, \alpha}} \lambda \tilde{\gamma}_{s}(d u) \leqslant \frac{1}{\lambda} \int_{A_{\lambda, \alpha}} \rho_{\alpha}(u) \tilde{\gamma}_{s}(d u)=\frac{1}{\lambda}\left(\tilde{\gamma}_{s} \circ \mathscr{G}_{\alpha}\right)\left(A_{\lambda, \alpha}\right) \lesssim \frac{1}{\lambda} \tilde{\gamma}_{s}\left(A_{\lambda, \alpha}\right)^{1-\delta} .
$$

Consequently

Finally, we write

$$
\tilde{\gamma}_{s}\left(A_{\lambda, \alpha}\right) \lesssim\left(\frac{1}{\lambda}\right)^{1 / \delta}
$$

$$
\left\|\rho_{\alpha}\right\|_{p}^{p}=p \int_{0}^{\infty} \lambda^{p-1} \tilde{\gamma}_{s}\left(A_{\lambda, \alpha}\right) d \lambda<\infty
$$

thanks to $(3.32)$ if $\delta^{-1}-(p-1)>1$, that is $p<\delta^{-1}$. The statement follows letting $p_{0}:=\delta^{-1}$. Indeed, recalling the definition (3.29) of $\delta$ (in particular $\delta \in(0,1)$ ) and the fact that $R^{*}$ only depends on $R$, we have $p_{0}=p_{0}(|\alpha|, R)>1$, as claimed.

\section{The Jacobian Determinant}

We denote the divergence operator div when applied to an $n$-th dimensional vectorial function $H: E_{N} \times E_{N} \mapsto \mathbb{C}$ as

$$
\operatorname{div} H\left(P_{N} u, P_{N} \bar{u}\right)=\sum_{|n| \leqslant N}\left(\frac{\partial H_{n}}{\partial u(n)}+\frac{\partial \bar{H}_{n}}{\partial \bar{u}(n)}\right)
$$

Let us recall Proposition 6.6 of [12].

Proposition 4.1. We have

$$
\operatorname{det}\left[\left(D P_{N} \mathscr{G}_{\alpha}^{N}\right)(u)\right]=\exp \left(\int_{0}^{\alpha} d \alpha^{\prime} \operatorname{div} i P_{N}\left(\mathcal{I}\left[P_{N} \mathscr{G}_{\alpha^{\prime}}^{N}(u)\right] P_{N} \mathscr{G}_{\alpha^{\prime}}^{N}(u)\right)\right) .
$$

Thus

We set for $s^{\prime}>0$ and $n_{0} \in \mathbb{N}$

$$
\left.\left.\frac{d}{d \alpha} \operatorname{det}\left(D \mathscr{G}_{\alpha}^{N}\right)(u)\right|_{\alpha=0}=i \operatorname{div} P_{N}\left(\mathcal{I}\left[P_{N} u\right] P_{N} u\right)\right)
$$

$$
L_{s^{\prime}, n_{0}}=L_{s^{\prime}, n_{0}}[u]:=\sup _{n \geqslant n_{0}}\left(n^{s^{\prime}}|u(n)|\right) .
$$


Lemma 4.2. For all $s^{\prime} \in[0, s)$ there is a $C_{s, s^{\prime}}>0$ such that

$$
\begin{aligned}
& \gamma_{s}\left(L_{s^{\prime}, n_{0}} \geqslant t\right) \leqslant C_{s, s^{\prime}} e^{-\frac{t^{2}}{2} n_{0}^{2\left(s-s^{\prime}\right)}} \\
& \left\|L_{s^{\prime}, n_{0}}^{2}\right\|_{L^{p}\left(\gamma_{s}\right)} \leqslant \frac{C_{s, s^{\prime}} p}{n_{0}^{s-s^{\prime}}} .
\end{aligned}
$$

Proof. It suffices to prove (4.4), then (4.5) readily follows.

For $\theta>0$ a simple Gaussian integral gives

$$
E_{s}\left[e^{\theta n^{s^{\prime}}|u(n)|}\right]=\exp \left(\frac{\theta^{2}}{2} \frac{n^{2 s^{\prime}}}{1+n^{2 s}}\right) .
$$

Therefore by Markov inequality

$$
\gamma_{s}\left(n^{s^{\prime}}|u(n)| \geqslant t\right) \leqslant \exp \left(-\theta t+\frac{\theta^{2}}{2} \frac{n^{2 s^{\prime}}}{1+n^{2 s}}\right)
$$

for any $\theta>0$ and in particular picking $\theta=t\left(1+n^{2 s}\right) n^{-2 s^{\prime}}$ we have

$$
\gamma_{s}\left(n^{s^{\prime}}|u(n)| \geqslant t\right) \leqslant \exp \left(-t^{2}\left(1+n^{2 s}\right) / 2 n^{2 s^{\prime}}\right) .
$$

Thereby by union bound

$$
\gamma_{s}\left(L_{s^{\prime}, n_{0}} \geqslant t\right) \leqslant \sum_{|n| \geqslant n_{0}} \exp \left(-t^{2}\left(1+n^{2 s}\right) / 2 n^{2 s^{\prime}}\right)<\frac{C}{s-s^{\prime}} e^{-\frac{t^{2}}{2} n_{0}^{2\left(s-s^{\prime}\right)}},
$$

where $C$ is uniformly bounded for $n_{0} \in \mathbb{N}$. Then (4.4) follows.

Lemma 4.3. Let $s>\frac{1}{2}, s^{\prime} \in\left(\frac{1}{2}, s\right), \varepsilon \in\left(0, \frac{1}{2}\right)$. The following bound holds

$$
\left.\mid \operatorname{div} P_{N}\left(\mathcal{I}\left[P_{N} u\right] P_{N} u\right)\right) \mid \lesssim \frac{\left\|P_{N} u\right\|_{L^{2}}^{2}}{N^{1-\varepsilon}}+L_{s^{\prime},\left\lfloor N^{\varepsilon}\right\rfloor}^{2} \frac{\log 2 N}{N^{\varepsilon\left(2 s^{\prime}-1\right)}}
$$

Proof. A direct computation from (1.2) yields

$$
\left(\mathcal{I}\left[P_{N} u\right]\right)(0)=0, \quad\left(\mathcal{I}\left[P_{N} u\right]\right)(m)=-\frac{i}{m} \sum_{|\ell|,|\ell-m| \leqslant N} u(\ell) \bar{u}(\ell-m) \quad \text { if } \quad m \neq 0,
$$

thus

$$
i\left(\mathcal{I}\left[P_{N} u\right] P_{N} u\right)(n)=\sum_{m: m \neq 0,|n-m| \leqslant N} \frac{1}{m} \sum_{\ell:|\ell|,|\ell-m|, \leqslant N} u(n-m) u(\ell) \bar{u}(\ell-m)
$$

and

$$
\begin{aligned}
\left.\operatorname{div} i P_{N}\left(\mathcal{I}\left[P_{N} u\right] P_{N} u\right)\right) & =2 \sum_{|n| \leqslant N} \sum_{m: m \neq 0,|n-m| \leqslant N} \frac{1}{m}|u(n-m)|^{2} \\
& =2 \sum_{n=1}^{N}\left(|u(-n)|^{2}-|u(n)|^{2}\right) \sum_{m=N-n+1}^{N+n} \frac{1}{m} .
\end{aligned}
$$

We pick any $\varepsilon \in\left(0, \frac{1}{2}\right)$ and split the sum in $1 \leqslant n \leqslant N^{\varepsilon}$ and $n>N^{\varepsilon}$. For the first part we notice

$$
\sum_{m=N-n+1}^{N+n} \frac{1}{m} \lesssim \ln (N+n)-\ln (N-n+1)=\ln \left(1+\frac{2 n-1}{N-n+1}\right)
$$


so that we have

$$
\left|\sum_{n=1}^{\left\lfloor N^{\varepsilon}\right\rfloor}\left(|u(-n)|^{2}-|u(n)|^{2}\right) \sum_{m=N-n+1}^{N+n} \frac{1}{m}\right| \lesssim\|u\|_{L^{2}}^{2} \ln \left(1+\frac{2 N^{\varepsilon}-1}{N-N^{\varepsilon}+1}\right) \lesssim \frac{\left\|P_{N} u\right\|_{L^{2}}^{2}}{N^{1-\varepsilon}} .
$$

For $n>N^{\varepsilon}$ we use $|u(n)|^{2} \leqslant n^{-2 s^{\prime}} L_{s^{\prime},\left\lfloor N^{\varepsilon}\right\rfloor}^{2}$ and (4.9) to estimate the modulus the second part of the sum as (remember $s^{\prime}>1 / 2$ )

$L_{s^{\prime},\left\lfloor N^{\varepsilon}\right\rfloor}^{2} \sum_{n=\left\lfloor N^{\varepsilon}\right\rfloor+1}^{N} \frac{1}{n^{2 s^{\prime}}} \ln \left(1+\frac{2 n-1}{N-n+1}\right) \leqslant \ln (2 N) L_{s^{\prime},\left\lfloor N^{\varepsilon}\right\rfloor}^{2} \sum_{n=\left\lfloor N^{\varepsilon}\right\rfloor+1}^{N} \frac{1}{n^{2 s^{\prime}}} \leqslant \frac{\ln 2 N}{N^{\left(2 s^{\prime}-1\right) \varepsilon}} L_{s^{\prime},\left\lfloor N^{\varepsilon}\right\rfloor}^{2}$.

This completes the proof of (4.6).

Therefore the following result is easily proven:

Proposition 4.4. Let $R>0, \varepsilon \in\left(0, \frac{1}{2}\right)$ small enough. There is $c(R)>0$ such that

$$
\left.\| 1_{\left\{\|u\|_{L^{2}} \leqslant R\right\}} \operatorname{div} P_{N}\left(\mathcal{I}\left[P_{N} u\right] P_{N} u\right)\right) \|_{L^{p}\left(\gamma_{s}\right)} \lesssim p \frac{c(R)}{N^{\varepsilon}} .
$$

\section{5. $L^{2}\left(\gamma_{s}\right)$-CONVERGENCE}

We start by a useful representation formula for

$$
F_{N}:=F\left[P_{N} u\right]:=\left.\frac{d}{d \alpha}\left\|\mathscr{G}_{\alpha} P_{N} u\right\|_{\dot{H}^{s}}^{2}\right|_{\alpha=0} .
$$

Lemma 5.1. Let $s>0, N \in \mathbb{N}$ and $u \in L^{2}(\mathbb{T})$. We have

$$
F_{N}=F_{N}^{<}+F_{N}^{\geqslant}
$$

where

$$
\begin{aligned}
& F_{N} \geqslant:=F \geqslant\left[P_{N} u\right]:=2 \operatorname{Re}\left(\sum_{\substack{\left|m_{1,2}\right|,\left|n_{1,2}\right| \leqslant N \\
n_{1}-m_{1} \neq 0 \\
\left|n_{1}-m_{1}\right| \geqslant \min \left(\left|n_{1}\right|,\left|m_{1}\right|\right) \\
n_{1}+n_{2}=m_{1}+m_{2}}} \frac{\left|m_{1}\right|^{2 s}}{m_{1}-n_{1}} u\left(n_{1}\right) u\left(n_{2}\right) \bar{u}\left(m_{1}\right) \bar{u}\left(m_{2}\right)\right) \\
& F_{N}^{<}:=F^{<}\left[P_{N} u\right] \\
& :=-2 \sum_{k \geqslant 1} \frac{(s)_{k}}{k !} \operatorname{Re}\left(\sum_{\substack{\left|m_{1,2}\right|,\left|n_{1,2}\right| \leqslant N \\
n_{1}-m_{1} \neq 0 \\
\left|n_{1}-m_{1}\right|<\min \left(\left|n_{1}\right|,\left|m_{1}\right|\right) \\
n_{1}+n_{2}=m_{1}+m_{2}}} \frac{\left(m_{1}-n_{1}\right)^{k-1}\left|m_{1}\right|^{k}}{\left|m_{1} n_{1}\right|^{k-s}} u\left(n_{1}\right) u\left(n_{2}\right) \bar{u}\left(m_{1}\right) \bar{u}\left(m_{2}\right)\right) .
\end{aligned}
$$

Proof. We use for $s>0$ the Taylor series converging for $|x|<1$

$$
(1+x)^{s}=\sum_{k \geqslant 0} \frac{(s)_{k}}{k !} x^{k}
$$

where $(s)_{k}$ denotes the falling factorial

$$
(s)_{0}=1, \quad(s)_{k}:=\prod_{j=0}^{k-1}(s-j), \quad k \geqslant 1
$$


Let now compute

$$
\begin{aligned}
\left(\mathscr{G}_{\alpha} P_{N} u\right)(n) & =1_{[-N, N]}(n) u(n)+\sum_{k \geqslant 1} \frac{(i \alpha)^{k}}{k !}\left(\left(\mathcal{I}\left[P_{N} u\right]\right)^{k} u\right)(n) \\
& :=u(n)+i \alpha \sum_{\left|n_{1}\right| \leqslant N, n-p=n_{1}} u\left(n_{1}\right)\left(\mathcal{I}\left[P_{N} u\right]\right)(p)+\sum_{k \geqslant 2} \frac{\alpha^{k}}{k !} r(k, n) .
\end{aligned}
$$

Using an integration by parts in the definition of the Fourier coefficient $\left(\left(\mathcal{I}\left[P_{N} u\right]\right)^{k} u\right)(n)$, we obtain

$$
|r(k, n)| \leqslant C\langle n\rangle^{-10}\left\|\left(\mathcal{I}\left[P_{N} u\right]\right)^{k} P_{N} u\right\|_{H^{10}} \leqslant C\langle n\rangle^{-10}\left\|P_{N} u\right\|_{H^{10}}^{2 k+1}
$$

Therefore, for $\alpha$ small enough, we get the estimate

$$
\left|\left\|\mathscr{G}_{\alpha} P_{N} u\right\|_{\dot{H}^{s}}^{2}-\left\|P_{N} u\right\|_{\dot{H}^{s}}^{2}-2 \alpha \operatorname{Im}\left(\sum_{\substack{\left|m_{1}, n_{1}\right| \leqslant N \\ m_{1}-p=n_{1}}}\left|m_{1}\right|^{2 s} \bar{u}\left(m_{1}\right) u\left(n_{1}\right) \mathcal{I}\left[P_{N} u\right](p)\right)\right| \leqslant C_{\left\|P_{N} u\right\|_{H^{10}}} \alpha^{2} .
$$

Thus

$$
\left.\frac{d}{d \alpha}\left\|\mathscr{G}_{\alpha}^{N} u\right\|_{\dot{H}^{s}}^{2}\right|_{\alpha=0}=\left.\frac{d}{d \alpha}\left\|\mathscr{G}_{\alpha} P_{N} u\right\|_{\dot{H}^{s}}^{2}\right|_{\alpha=0}=2 \operatorname{Im}\left(\sum_{\substack{\left|m_{1}, n_{1}\right| \leqslant N \\ m_{1}-p=n_{1}}}\left|m_{1}\right|^{2 s} \bar{u}\left(m_{1}\right) u\left(n_{1}\right) \mathcal{I}\left[P_{N} u\right](p)\right) .
$$

Now we conveniently represent

$$
\left.\frac{d}{d \alpha}\left\|\mathscr{G}_{\alpha} P_{N} u\right\|_{\dot{H}^{s}}^{2}\right|_{\alpha=0}=F_{N}^{<}+F_{N}^{\geqslant},
$$

where

$$
\begin{gathered}
F_{N}^{\geqslant}:=2 \operatorname{Im}\left(\sum_{\substack {\left|m_{1}, n_{1}\right| \leqslant N \\
|p| \geqslant \begin{subarray}{c}{\min \left(\left|n_{1}\right|,\left|m_{1}\right|\right) \\
m_{1}-p=n_{1}{ | m _ { 1 } , n _ { 1 } | \leqslant N \\
| p | \geqslant \begin{subarray} { c } { \operatorname { m i n } ( | n _ { 1 } | , | m _ { 1 } | ) \\
m _ { 1 } - p = n _ { 1 } } }\end{subarray}}\left|m_{1}\right|^{2 s} \bar{u}\left(m_{1}\right) u\left(n_{1}\right) \mathcal{I}\left[P_{N} u\right](p)\right) \\
F_{N}^{<}:=2 \operatorname{Im}\left(\sum_{\substack{\left|m_{1}, n_{1}\right| \leqslant N, m_{1}-p=n_{1} \\
|p|<\min \left(\left|n_{1}\right|,\left|m_{1}\right|\right)}}\left|m_{1}\right|^{s}\left|n_{1}+p\right|^{s} \bar{u}\left(m_{1}\right) u\left(n_{1}\right) \mathcal{I}\left[P_{N} u\right](p)\right) .
\end{gathered}
$$

Then (5.3) is easily obtained from (5.7) by using

$$
\left(\mathcal{I}\left[P_{N} u\right]\right)(p)= \begin{cases}0 & \text { if } p=0 \\ -\frac{i}{p} \sum_{\substack{\left|n_{2}\right|,\left|m_{2}\right| \leqslant N \\ p=n_{2}-m_{2}}} u\left(n_{2}\right) \bar{u}\left(m_{2}\right) & \text { if } p \neq 0\end{cases}
$$

Let us look at $F_{N}^{<}$. Using (5.5) we have

$$
\begin{aligned}
& F_{N}^{<}=2 \operatorname{Im}\left(\sum_{\substack{\left|m_{1}, n_{1}\right| \leqslant N, m_{1}-p=n_{1} \\
|p|<\min \left(\left|n_{1}\right|,\left|m_{1}\right|\right)}}\left|m_{1}\right|^{s}\left|n_{1}\right|^{s} \sum_{k \geqslant 0} \frac{(s)_{k}}{k !} \frac{p^{k}}{\left|n_{1}\right|^{k}} \bar{u}\left(m_{1}\right) u\left(n_{1}\right) \mathcal{I}\left[P_{N} u\right](p)\right) \\
& =2 \operatorname{Im}\left(\sum_{\substack{\left|m_{1}, n_{1}\right| \leqslant N, m_{1}-p=n_{1} \\
|p|<\min \left(\left|n_{1}\right|,\left|m_{1}\right|\right)}}\left|m_{1}\right|^{s}\left|n_{1}\right|^{s} \sum_{k \geqslant 1} \frac{(s)_{k}}{k !} \frac{p^{k}}{\left|n_{1}\right|^{k}} \bar{u}\left(m_{1}\right) u\left(n_{1}\right) \mathcal{I}\left[P_{N} u\right](p)\right)
\end{aligned}
$$

as $\mathcal{I}[u](p)=\overline{\mathcal{I}[u](-p)}$ yields

$$
\operatorname{Im}\left(\sum_{\substack{\left|m_{1}, n_{1}\right| \leqslant N, m_{1}-p=n_{1} \\|p|<\min \left(\left|n_{1}\right|,\left|m_{1}\right|\right)}}\left|m_{1}\right|^{s}\left|n_{1}\right|^{s} \bar{u}\left(m_{1}\right) u\left(n_{1}\right) \mathcal{I}\left[P_{N} u\right](p)\right)=0 .
$$

When we plug (5.9) in (5.10) we obtain (5.4). 
Remark 5.2. By the same argument we also have

$$
F_{N}=2 \operatorname{Re}\left(\sum_{\substack{\left|m_{1,2}\right|\left|n_{1,2}\right| \leqslant N \\ n_{1}+n_{2}=m_{1}+m_{2} \\ n_{1}-m_{1} \neq 0}} \frac{\left|m_{1}\right|^{2 s}}{m_{1}-n_{1}} u\left(n_{1}\right) u\left(n_{2}\right) \bar{u}\left(m_{1}\right) \bar{u}\left(m_{2}\right)\right) .
$$

In what follows we shall use the Wick formula for expectation values of multilinear forms of Gaussian random variables in the following form. Let $\ell \in \mathbb{N}$ and $S_{\ell}$ be the symmetric group on $\{1, \ldots, \ell\}$, whose elements are denoted by $\sigma$. Recalling (1.5) we have

$$
\begin{aligned}
E_{s}\left[\prod_{j=1}^{\ell} u\left(n_{j}\right) \bar{u}\left(m_{j}\right)\right] & =\sum_{\sigma \in S_{\ell}} \prod_{j=1}^{\ell} \frac{\delta_{m_{j}, n_{\sigma(j)}}}{1+\left|n_{j}\right|^{2 s}} \\
& \simeq \sum_{\sigma \in S_{\ell}} \prod_{j=1}^{\ell} \frac{\delta_{m_{j}, n_{\sigma(j)}}}{\left\langle n_{j}\right\rangle^{2 s}},
\end{aligned}
$$

where $\langle\cdot\rangle=\left(1+|\cdot|^{2}\right)^{1 / 2}$. We convey that the labels $m_{i}$ (respectively $\left.n_{i}\right)$ are associated to the Fourier coefficients of $\bar{u}$ (respectively $u$ ). We say that $\sigma$ contracts the pairs of indexes $\left(m_{j}, n_{\sigma(j)}\right)$ and we shorten for any $\Omega \subset \mathbb{Z}^{\ell} \times \mathbb{Z}^{\ell}$

$$
\sigma(\Omega):=\Omega \cap\left\{m_{i}=n_{\sigma(i)}, i=1, \ldots, \ell\right\}, \quad \sigma \in S_{\ell},
$$

We also define the set $\bar{\Omega}$ to be obtained by $\Omega$ swapping the role of $n_{i}$ and $m_{i} i=1, \ldots \ell$.

The following elementary bound will be useful in the proof of the forthcoming Lemma 5.5.

Lemma 5.3. Let $s>\frac{1}{2}$. Then

$$
\sum_{q \in \mathbb{Z}} \frac{1}{\langle p-q\rangle\langle q\rangle^{2 s}}=\sum_{q \in \mathbb{Z}} \frac{1}{\langle p-q\rangle^{2 s}\langle q\rangle} \leqslant \frac{C}{\langle p\rangle},
$$

for a constant $C$ which only depends on s. Similarly

$$
\sum_{q \in \mathbb{Z}} \frac{1}{\left\langle p-q^{2}\right\rangle\langle q\rangle^{2 s}} \leqslant \frac{C}{\langle p\rangle} .
$$

Proof. The identity in (5.14) is just a change of variables. We just show (5.14), namely

$$
\sum_{q \in \mathbb{Z}} \frac{1}{\langle p-q\rangle^{2 s}\langle q\rangle} \leqslant \frac{C}{\langle p\rangle}
$$

being the proof of (5.15) almost identical. First we notice that

$$
\sum_{q \in \mathbb{Z}:|q| \geqslant \frac{|p|}{2}} \frac{1}{\langle p-q\rangle^{2 s}\langle q\rangle} \leqslant \frac{2}{\langle p\rangle} \sum_{q \in \mathbb{Z}} \frac{1}{\langle p-q\rangle^{2 s}} \leqslant \frac{C}{\langle p\rangle} .
$$

Then we notice that for $|q|<\frac{|p|}{2}$ we have by triangle inequality $|p-q| \geqslant|p|-|q|>\frac{|p|}{2}$, so that

$$
\begin{aligned}
\sum_{q \in \mathbb{Z}:|q|<\frac{|p|}{2}} & \frac{1}{\langle p-q\rangle^{2 s}\langle q\rangle} \leqslant \frac{2}{\langle p\rangle} \sum_{q \in \mathbb{Z}} \frac{1}{\langle p-q\rangle^{2 s-1}} \frac{1}{\langle q\rangle} \\
& \leqslant \frac{2}{\langle p\rangle}\left(\sum_{q \in \mathbb{Z}} \frac{1}{\langle p-q\rangle^{2 s}}\right)^{\frac{2 s-1}{2 s}}\left(\sum_{q \in \mathbb{Z}} \frac{1}{\langle q\rangle^{2 s}}\right)^{\frac{1}{2 s}} \leqslant \frac{C}{\langle p\rangle},
\end{aligned}
$$

where we used Hölder inequality with the conjugate pair $\frac{2 s}{2 s-1}, 2 s$ which concludes the proof. 
Lemma 5.4. Let $\varepsilon \in(0,1)$. Then

$$
\sum_{|q| \in \mathbb{Z}, q \neq p} \frac{1}{\left\langle q^{2}-p^{2}\right\rangle} \leqslant \frac{C_{\varepsilon}}{\langle p\rangle^{1-\varepsilon}}
$$

Proof. It is clearly sufficient to show that for all $p \in \mathbb{N} \backslash\{0\}$ we have

$$
\sum_{q \geqslant 1, q \neq p} \frac{1}{\left\langle q^{2}-p^{2}\right\rangle} \leqslant \frac{C_{\varepsilon}}{p^{1-\varepsilon}}
$$

We split the sum over $q=1, \ldots, p-1$ and $q \geqslant p+1$. In the first case we estimate

$$
\sum_{q=1}^{p-1} \frac{1}{\left\langle q^{2}-p^{2}\right\rangle} \lesssim \sum_{q=1}^{p-1} \frac{1}{p^{2}-q^{2}}=\sum_{q=0}^{p-1} \frac{1}{2 p}\left(\frac{1}{p-q}+\frac{1}{p+q}\right) \lesssim \frac{1}{p} \sum_{q=1}^{2 p-1} \frac{1}{q} \lesssim \frac{\ln (2 p)}{p}
$$

In the second case we estimate

$$
\begin{aligned}
\sum_{q \geqslant p+1} \frac{1}{\left\langle q^{2}-p^{2}\right\rangle} & \lesssim \sum_{q \geqslant p+1} \frac{1}{q^{2}-p^{2}}=\sum_{q \geqslant p+1} \frac{1}{2 q}\left(\frac{1}{q-p}+\frac{1}{q+p}\right) \\
& \leqslant \frac{1}{p^{1-\varepsilon}} \sum_{q \geqslant p+1} \frac{1}{q^{\varepsilon}}\left(\frac{1}{q-p}+\frac{1}{q+p}\right) \\
& \leqslant \frac{1}{p^{1-\varepsilon}}\left(\sum_{q \geqslant 1} \frac{1}{q^{1+\varepsilon}}\right)^{\frac{\varepsilon}{1+\varepsilon}}\left(\sum_{q \geqslant p+1}\left(\frac{1}{q-p}+\frac{1}{q+p}\right)^{1+\varepsilon}\right)^{\frac{1}{1+\varepsilon}}
\end{aligned}
$$

where we used Hölder inequality with the conjugate pair $\frac{1+\varepsilon}{\varepsilon}, 1+\varepsilon$. Of course the second factor in the above formula is finite for any $\varepsilon>0$. The third factor is also finite as

$$
\left(\sum_{q \geqslant p+1}\left(\frac{1}{q-p}+\frac{1}{q+p}\right)^{1+\varepsilon}\right)^{\frac{1}{1+\varepsilon}} \leqslant C_{\varepsilon}\left(\sum_{q \geqslant 1}\left(\frac{1}{q}\right)^{1+\varepsilon}+\sum_{q \geqslant 1+p}\left(\frac{1}{q+p}\right)^{1+\varepsilon}\right)^{\frac{1}{1+\varepsilon}}
$$

which is bounded for $\varepsilon>0$. We conclude

$$
(5.20) \leqslant \frac{C_{\varepsilon}}{p^{1-\varepsilon}}
$$

which ends the proof.

The following lemma is crucial for the rest of the paper. The idea behind is that exploiting the cancellations induced by the randomness one can recover the $\varepsilon$-derivative loss of $F_{N}$. Since we deal with a Gaussian space, it suffices to do that in $L^{2}$. The $L^{2}$ bound is achieved using the Wick formula (1.5) for expectation values of multilinear forms of Gaussian random variables. Another important tool used in the proof (to handle the permutations (f)-(g)-(h) in (5.26)) is the decomposition (5.1). The idea of this decomposition is to treat separately the low and high Fourier modes of $\mathcal{I}[u]$. For the high Fourier modes we take advantage of the regularisation given by the fact that $\mathcal{I}[u]$ is an anti-derivative. For the low Fourier modes we used the cancellation (5.11) in order to eliminate the first term (corresponding to $k=1$ ) of the Taylor expansion (5.10), which is the less regular.

Lemma 5.5. Let $s>1 / 2$ and $M \in \mathbb{N}$. Then $F_{M} \rightarrow F$ in $L^{2}\left(\gamma_{s}\right)$ as $M \rightarrow \infty$, with

$$
\left\|F_{M}-F\right\|_{L^{2}\left(\gamma_{s}\right)} \lesssim \frac{1}{M^{\min \left(\frac{1}{2}, s-\frac{1}{2}\right)}}
$$


Proof. Let $N>M$ and define for $a, b \in \mathbb{N}$

$$
\begin{gathered}
A_{N, M}^{a, b}:=\left\{\left|n_{a, b}\right|,\left|m_{a, b}\right| \leqslant N,\left|n_{a}-m_{a}\right| \geqslant \min \left(\left|n_{a}\right|,\left|m_{a}\right|\right), n_{a} \neq m_{a},\right. \\
\left.n_{a}+n_{b}=m_{a}+m_{b}, \max \left(\left|m_{a, b}\right|,\left|n_{a, b}\right|\right)>M\right\} .
\end{gathered}
$$

We have

$$
F_{N}-F_{M}=2 \operatorname{Re}\left(\sum_{A_{N, M}^{1,2}} \frac{\left|m_{1}\right|^{2 s}}{m_{1}-n_{1}} \bar{u}\left(m_{1}\right) \bar{u}\left(m_{2}\right) u\left(n_{1}\right) u\left(n_{2}\right)\right)
$$

Now we square

$$
\left|F_{N}(u)-F_{M}(u)\right|^{2}=4 \sum_{A_{N, M}^{1,2} \times A_{N, M}^{3,4}} \frac{\left|m_{1}\right|^{2 s}}{m_{1}-n_{1}} \frac{\left|n_{3}\right|^{2 s}}{n_{3}-m_{3}} \prod_{j=1}^{4} \bar{u}\left(m_{j}\right) u\left(n_{j}\right)
$$

and take the expected value w.r.t. $\gamma_{s}$ using formula (5.13) with $\ell=4$

$$
\begin{aligned}
\frac{1}{4}\left\|F_{N}-F_{M}\right\|_{L^{2}\left(\gamma_{s}\right)}^{2} & =\sum_{A_{N, M}^{1,2} \times A_{N, M}^{3,4}} \frac{\left|m_{1}\right|^{2 s}}{m_{1}-n_{1}} \frac{\left|n_{3}\right|^{2 s}}{n_{3}-m_{3}} E_{s}\left[\prod_{j=1}^{4} \bar{u}\left(m_{j}\right) u\left(n_{j}\right)\right] \\
& =\sum_{A_{N, M}^{1,2} \times A_{N, M}^{3,4}} \frac{m_{1}^{2 s}}{m_{1}-n_{1}} \frac{n_{3}^{2 s}}{n_{3}-m_{3}} \sum_{\sigma \in S_{4}} \prod_{j=1}^{4} \frac{\delta_{m_{j}, n_{\sigma(j)}}}{\left\langle n_{j}\right\rangle^{2 s}}
\end{aligned}
$$

Therefore

$$
(5.24)=\sum_{\sigma \in S_{4}} \sum_{\sigma\left(A_{N, M}^{1,2} \times A_{N, M}^{3,4}\right)} \frac{\left|n_{\sigma(1)}\right|^{2 s}}{n_{\sigma(1)}-n_{1}} \frac{\left|n_{3}\right|^{2 s}}{n_{3}-n_{\sigma(3)}} \prod_{j=1}^{4} \frac{1}{\left\langle n_{j}\right\rangle^{2 s}}
$$

We note that contractions with $\sigma(j)=j$ for some $j \in\{1, \ldots, 4\}$ yields $\sigma\left(A_{N, M}^{1,2} \times A_{N, M}^{3,4}\right)=\emptyset$. Therefore the sum over $\sigma$ runs actually in

$$
\left.S_{4}^{\prime}:=\left\{\sigma \in S_{4}: \sigma(j) \neq j, \forall j=1, \ldots, 4\right\}\right),
$$

which contains 9 elements. Moreover the contribution of $\sigma=(2,1,4,3)$ to $(5.25)$ is

$$
\sum_{\substack{\left.\left|n_{i}\right| \leqslant N, i=1, \ldots, 4 \\
n_{2} \neq n_{1}, n_{4} \neq n_{3} \\
n_{1},\left|n_{2}\right|\right), \max \left(\left|n_{3}\right|,\left|n_{4}\right|\right)>M}} \frac{\left|n_{2}\right|^{2 s}}{n_{2}-n_{1}} \frac{\left|n_{3}\right|^{2 s}}{n_{4}-n_{3}}=\left(\sum_{\begin{array}{c}
\left|n_{1}\right|,\left|n_{2}\right| \leqslant N \\
n_{2} \neq n_{1} \\
\max \left(\left|n_{1}\right|,\left|n_{2}\right|\right) \geqslant M
\end{array}} \frac{\left|n_{2}\right|^{2 s}}{n_{2}-n_{1}}\right)^{2},
$$

which is zero, due to its antisymmetry w.r.t. $n_{1} \mapsto-n_{1}, n_{2} \mapsto-n_{2}$. It remains to consider the following 8 permutations:

$$
\sigma= \begin{cases}(a) & (3,1,4,2) \\ (b) & (4,1,2,3) \\ (c) & (2,3,4,1) \\ (d) & (2,4,1,3) \\ (e) & (4,3,2,1) \\ (f) & (3,4,2,1) \\ (g) & (3,4,1,2) \\ (h) & (4,3,1,2)\end{cases}
$$


- Case (a), $\sigma=(3,1,4,2)$. Note that $n_{1}+n_{2}=n_{\sigma(1)}+n_{\sigma(2)}$ and $n_{3}+n_{4}=n_{\sigma(3)}+n_{\sigma(4)}$ reduces to $n_{2}=n_{3}$, so we need to evaluate

$$
\begin{aligned}
& \left|\sum_{\substack{\left|n_{i}\right| \leqslant N, i=1,2,4 \\
n_{2} \neq n_{1}, n_{4} \neq n_{2} \\
\max \left(\left|n_{1}\right|,\left|n_{2}\right|\right), \max \left(\left|n_{2}\right|,\left|n_{4}\right|\right)>M}} \frac{1}{\left(n_{2}-n_{1}\right)\left(n_{2}-n_{4}\right)\left\langle n_{1}\right\rangle^{2 s}\left\langle n_{4}\right\rangle^{2 s}}\right| \\
& \lesssim \sum_{\substack{\left|n_{i}\right| \leqslant N, i=1,2,4 \\
\max \left(\left|n_{1}\right|,\left|n_{2}\right|\right), \max \left(\left|n_{2}\right|,\left|n_{4}\right|\right)>M}} \frac{1}{\left\langle n_{2}-n_{1}\right\rangle\left\langle n_{2}-n_{4}\right\rangle\left\langle n_{1}\right\rangle^{2 s}\left\langle n_{4}\right\rangle^{2 s}}
\end{aligned}
$$

If $\left|n_{2}\right|>M$, taking advantage of the symmetry w.r.t. $n_{1} \leftrightarrow n_{4}$, we get

$$
\left.(5.27)\right|_{\left|n_{2}\right|>M} \lesssim \sum_{M<\left|n_{2}\right| \leqslant N}\left(\sum_{\left|n_{4}\right| \leqslant N} \frac{1}{\left\langle n_{2}-n_{4}\right\rangle} \frac{1}{\left\langle n_{4}\right\rangle^{2 s}}\right)^{2} \lesssim \sum_{M \leqslant\left|n_{2}\right| \leqslant N} \frac{1}{\left\langle n_{2}\right\rangle^{2}} \lesssim \frac{1}{M}
$$

where we used (5.14) in the second inequality. Otherwise it has to be $\left|n_{1}\right|>M$, so that

$$
\begin{aligned}
\left.(5.27)\right|_{\left|n_{1}\right|>M} & \lesssim \sum_{\substack{M<\left|n_{1}\right| \leqslant N \\
\left|n_{4}\right| \leqslant N}} \frac{1}{\left\langle n_{1}\right\rangle^{2 s}} \frac{1}{\left\langle n_{4}\right\rangle^{2 s}}\left(\sum_{\left|n_{2}\right| \leqslant N} \frac{1}{\left\langle n_{2}-n_{1}\right\rangle} \frac{1}{\left\langle n_{2}-n_{4}\right\rangle}\right) \\
& \leqslant \sum_{\substack{M<\left|n_{1}\right| \leqslant N \\
\left|n_{4}\right| \leqslant N}} \frac{1}{\left\langle n_{1}\right\rangle^{2 s}} \frac{1}{\left\langle n_{4}\right\rangle^{2 s}}\left(\sum_{\left|n_{2}\right| \leqslant 2 N} \frac{1}{\left\langle n_{2}+n_{1}-n_{4}\right\rangle} \frac{1}{\left\langle n_{2}\right\rangle}\right) \\
& \lesssim \sum_{\substack{M<\left|n_{1}\right| \leqslant N \\
\left|n_{4}\right| \leqslant N}} \frac{1}{\left\langle n_{1}\right\rangle^{2 s}} \frac{1}{\left\langle n_{4}\right\rangle^{2 s}} \frac{1}{\left\langle n_{1}-n_{4}\right\rangle} \lesssim \sum_{M<\left|n_{1}\right| \leqslant N} \frac{1}{\left\langle n_{1}\right\rangle^{2 s+1}} \lesssim \frac{1}{M^{2 s}},
\end{aligned}
$$

where we used (5.14) in the third inequality.

- Case (b), $\sigma=(4,1,2,3)$. Note that $n_{1}+n_{2}=n_{\sigma(1)}+n_{\sigma(2)}$ and $n_{3}+n_{4}=n_{\sigma(3)}+n_{\sigma(4)}$ reduce to $n_{2}=n_{4}$, so we need to evaluate

$$
\left|\sum_{\substack{\left|n_{i}\right| \leqslant N, i=1,2,3 \\ n_{2} \neq n_{1}, n_{3} \\ \max \left(\left|n_{1}\right|,\left|n_{2}\right|\right), \max \left(\left|n_{2}\right|,\left|n_{3}\right|\right)>M}} \frac{1}{\left(n_{2}-n_{1}\right)\left(n_{3}-n_{2}\right)\left\langle n_{1}\right\rangle^{2 s}\left\langle n_{2}\right\rangle^{2 s}}\right|
$$

If we restrict the sum also to $n_{3} \neq-n_{2}$, we ca can exploit the symmetry $n_{3} \mapsto-n_{3}$ to bound

$$
\begin{aligned}
\left.(5.29)\right|_{n_{3} \neq-n_{2}} & =\frac{1}{2}\left|\sum_{\substack{\left|n_{i}\right| \leqslant N, i=1,2,3 \\
n_{2} \neq n_{1},\left|n_{3}\right| \neq\left|n_{2}\right|}} \frac{1}{\left(n_{2}-n_{1}\right)} \frac{1}{\left\langle n_{1}\right\rangle^{2 s}} \frac{1}{\left\langle n_{2}\right\rangle^{2 s}}\left(\frac{1}{n_{3}-n_{2}}-\frac{1}{n_{3}+n_{2}}\right)\right| \\
\leqslant & \sum_{\substack{\left|n_{i}\right| \leqslant N, i=1,2,3 \\
\max \left(\left|n_{1}\right|,\left|n_{2}\right|\right), \max \left(\left|n_{2}\right|,\left|n_{3}\right|\right)>M}} \frac{1}{\left\langle n_{2}-n_{1}\right\rangle} \frac{1}{\left\langle n_{1}\right\rangle^{2 s}} \frac{1}{\left\langle n_{2}\right\rangle^{2 s}} \frac{\left|n_{2}\right|}{\left\langle n_{3}^{2}-n_{2}^{2}\right\rangle} .
\end{aligned}
$$

If $\left|n_{2}\right|>M$, using

$$
\sum_{\left|n_{3}\right| \leqslant N} \frac{1}{\left\langle n_{3}^{2}-n_{2}^{2}\right\rangle} \leqslant C
$$


we can bound

$$
\begin{aligned}
&\left.(5.29)\right|_{\substack{n_{3} \neq-n_{2} \\
\left|n_{2}\right|>M}} \lesssim \sum_{M<\left|n_{2}\right| \leqslant N} \frac{\left|n_{2}\right|}{\left\langle n_{2}\right\rangle^{2 s}}\left(\sum_{\left|n_{1}\right| \leqslant N} \frac{1}{\left\langle n_{2}-n_{1}\right\rangle\left\langle n_{1}\right\rangle^{2 s}}\right)\left(\sum_{\left|n_{3}\right| \leqslant N} \frac{1}{\left\langle n_{3}^{2}-n_{2}^{2}\right\rangle}\right) \\
& \lesssim \sum_{M<\left|n_{2}\right| \leqslant N} \frac{\left|n_{2}\right|}{\left\langle n_{2}\right\rangle^{2 s}}\left(\sum_{\left|n_{1}\right| \leqslant N} \frac{1}{\left\langle n_{2}-n_{1}\right\rangle\left\langle n_{1}\right\rangle^{2 s}}\right) \\
& \lesssim \sum_{M<\left|n_{2}\right| \leqslant N} \frac{1}{\left\langle n_{2}\right\rangle^{2 s}} \lesssim \frac{1}{M^{2 s-1}}
\end{aligned}
$$

where we used (5.14) in the third inequality. Otherwise it must be $\left|n_{3}\right|>M$, so

$$
\begin{aligned}
&\left.(5.29)\right|_{\substack{n_{3} \neq-n_{2} \\
\left|n_{3}\right|>M}} \lesssim \sum_{\substack{M<\left|n_{3}\right| \leqslant N \\
\left|n_{2}\right| \leqslant N}} \frac{\left|n_{2}\right|}{\left\langle n_{3}^{2}-n_{2}^{2}\right\rangle\left\langle n_{2}\right\rangle^{2 s}}\left(\sum_{\left|n_{1}\right| \leqslant N} \frac{1}{\left\langle n_{2}-n_{1}\right\rangle\left\langle n_{1}\right\rangle^{2 s}}\right) \\
& \lesssim \sum_{\substack{M<\left|n_{3}\right| \leqslant N \\
\left|n_{2}\right| \leqslant N}} \frac{1}{\left\langle n_{3}^{2}-n_{2}^{2}\right\rangle\left\langle n_{2}\right\rangle^{2 s}} \lesssim \sum_{M<\left|n_{3}\right| \leqslant N} \frac{1}{\left\langle n_{3}^{2}\right\rangle} \lesssim \frac{1}{M}
\end{aligned}
$$

where we used (5.14) in the second inequality and (5.15) in the third one. When we sum over $n_{3}=-n_{2}$ we get instead

$$
\left.(5.29)\right|_{n_{3}=-n_{2}} \lesssim \sum_{\substack{M<\left|n_{2}\right| \leqslant N \\\left|n_{1}\right| \leqslant N, n_{1} \neq n_{2}}} \frac{1}{\left\langle n_{2}-n_{1}\right\rangle} \frac{1}{\left\langle n_{1}\right\rangle^{2 s}} \frac{1}{\left\langle n_{2}\right\rangle^{2 s+1}} \lesssim \sum_{M<\left|n_{2}\right| \leqslant N} \frac{1}{\left\langle n_{2}\right\rangle^{2 s+1}} \lesssim \frac{1}{M^{2 s}}
$$

- Case (c), $\sigma=(2,3,4,1)$. Note that $n_{1}+n_{2}=n_{\sigma(1)}+n_{\sigma(2)}$ and $n_{3}+n_{4}=n_{\sigma(3)}+n_{\sigma(4)}$ reduce to $n_{1}=n_{3}$, so we need to evaluate

$$
\left|\sum_{\substack{\left|n_{i}\right| \leqslant N, i=1,2,4 \\ n_{1} \neq n_{2}, n_{4} \\ \max \left(\left|n_{1}\right|,\left|n_{2}\right|\right), \max \left(\left|n_{1}\right|,\left|n_{4}\right|\right)>M}} \frac{1}{\left(n_{2}-n_{1}\right)\left(n_{1}-n_{4}\right)\left\langle n_{1}\right\rangle^{2 s}\left\langle n_{4}\right\rangle^{2 s}}\right|
$$

that, modulo rename $\left(n_{1}, n_{4}, n_{2}\right)=\left(n_{2}^{\prime}, n_{1}^{\prime}, n_{3}^{\prime}\right)$, is the (5.29). So we proceed as in the case (b).

- Case $(\mathrm{d}), \sigma=(2,4,1,3)$. Note that $n_{1}+n_{2}=n_{\sigma(1)}+n_{\sigma(2)}$ and $n_{3}+n_{4}=n_{\sigma(3)}+n_{\sigma(4)}$ reduce to $n_{1}=n_{4}$, so we need to evaluate

$$
\left|\sum_{\substack{\left|n_{i}\right| \leqslant N, i=1,2,3 \\ n_{1} \neq n_{2}, n_{3} \\ \max \left(\left|n_{1}\right|,\left|n_{2}\right|\right), \max \left(\left|n_{1}\right|,\left|n_{3}\right|\right)>M}} \frac{1}{\left(n_{2}-n_{1}\right)\left(n_{3}-n_{1}\right)\left\langle n_{1}\right\rangle^{4 s}}\right|
$$


When we restrict the sum also over $n_{1} \neq-n_{2},-n_{3}$, we can exploit first the map $n_{3} \leftrightarrow-n_{3}$ and then $n_{1} \leftrightarrow-n_{1}$ to get

$$
\begin{aligned}
& \left.(5.32)\right|_{n_{1} \neq-n_{2},-n_{3}}=\left|\sum_{\substack{\left|n_{i}\right| \leqslant N, i=1,2,3 \\
\left|n_{1}\right| \neq\left|n_{2}\right|,\left|n_{3}\right|}} \frac{1}{\left(n_{2}-n_{1}\right)\left(n_{3}-n_{1}\right)} \frac{1}{\left\langle n_{1}\right\rangle^{4 s}}\right| \\
& =\frac{1}{2}\left|\quad \sum_{\substack{\left|n_{i}\right| \leqslant N, i=1,2,3 \\
\left|n_{1}\right| \neq\left|n_{2}\right|,\left|n_{3}\right|}} \frac{1}{\left(n_{2}-n_{1}\right)} \frac{1}{\left\langle n_{1}\right\rangle^{4 s}}\left(\frac{1}{\left(n_{3}-n_{1}\right)}-\frac{1}{\left(n_{3}+n_{1}\right)}\right)\right| \\
& \max \left(\left|n_{1}\right|,\left|n_{2}\right|\right), \max \left(\left|n_{1}\right|,\left|n_{3}\right|\right)>M \\
& =\left|\sum_{\substack{\left|n_{i}\right| \leqslant N, i=1,2,3 \\
\left|n_{1}\right| \neq\left|n_{2}\right|,\left|n_{3}\right|}} \frac{1}{\left(n_{2}-n_{1}\right)} \frac{1}{\left\langle n_{1}\right\rangle^{4 s}} \frac{n_{1}}{\left(n_{3}^{2}-n_{1}^{2}\right)}\right| \\
& \max \left(\left|n_{1}\right|,\left|n_{2}\right|\right), \max \left(\left|n_{1}\right|,\left|n_{3}\right|\right)>M \\
& =\frac{1}{2}\left|\sum_{\substack{\left|n_{i}\right| \leqslant N, i=1,2,3 \\
\left|n_{1}\right| \neq\left|n_{2}\right|,\left|n_{3}\right|}}\left(\frac{n_{1}}{\left(n_{2}-n_{1}\right)}-\frac{n_{1}}{\left(n_{2}+n_{1}\right)}\right) \frac{1}{\left\langle n_{1}\right\rangle^{4 s}} \frac{1}{\left(n_{3}^{2}-n_{1}^{2}\right)}\right| \\
& \max \left(\left|n_{1}\right|,\left|n_{2}\right|\right), \max \left(\left|n_{1}\right|,\left|n_{3}\right|\right)>M \\
& \lesssim \sum_{\substack{\left|n_{i}\right| \leqslant N, i=1,2,3 \\
\max \left(\left|n_{1}\right|,\left|n_{2}\right|\right), \max \left(\left|n_{1}\right|,\left|n_{3}\right|\right)>M}} \frac{1}{\left\langle n_{2}^{2}-n_{1}^{2}\right\rangle\left\langle n_{3}^{2}-n_{1}^{2}\right\rangle} \frac{1}{\left\langle n_{1}\right\rangle^{4 s-2}} .
\end{aligned}
$$

Thus if $\left|n_{1}\right|>M$ we have, for all $\varepsilon \in(0,1)$

$$
\begin{aligned}
&\left.(5.32)\right|_{\substack{n_{1} \neq-n_{2},-n_{3} \\
\left|n_{1}\right|>M}} \lesssim \sum_{M<\left|n_{1}\right| \leqslant N} \frac{1}{\left\langle n_{1}\right\rangle^{4 s-2}}\left(\sum_{\left|n_{2}\right| \leqslant N, n_{2} \neq n_{1}} \frac{1}{\left\langle n_{2}^{2}-n_{1}^{2}\right\rangle}\right)^{2} \\
& \leqslant \sum_{M<\left|n_{1}\right| \leqslant N} \frac{C_{\varepsilon}}{\left\langle n_{1}\right\rangle^{4 s-\varepsilon}} \lesssim \frac{C_{\varepsilon}}{M^{4 s-1-\varepsilon}},
\end{aligned}
$$

where we used the symmetry $n_{2} \leftrightarrow n_{3}$ and the inequality (5.17). Otherwise it has to be $\left|n_{2}\right|>M$, so that

$$
\begin{aligned}
&\left.(5.32)\right|_{\substack{n_{1} \neq-n_{2},-n_{3} \\
\left|n_{2}\right|>M}} \lesssim \sum_{\substack{M<\left|n_{2}\right| \leqslant N \\
\left|n_{1}\right| \leqslant N}} \frac{1}{\left\langle n_{2}^{2}-n_{1}^{2}\right\rangle\left\langle n_{1}\right\rangle^{4 s-2}} \sum_{\left|n_{3}\right| \leqslant N, n_{3} \neq n_{1}} \frac{1}{\left\langle n_{3}^{2}-n_{1}^{2}\right\rangle} \\
& \leqslant \sum_{\substack{M<\left|n_{2}\right| \leqslant N \\
\left|n_{1}\right| \leqslant N}} \frac{C_{\varepsilon}}{\left\langle n_{2}^{2}-n_{1}^{2}\right\rangle\left\langle n_{1}\right\rangle^{4 s-1-\varepsilon}} \leqslant \sum_{M<\left|n_{2}\right| \leqslant N} \frac{C_{\varepsilon}}{\left\langle n_{2}\right\rangle^{2}} \lesssim \frac{C_{\varepsilon}}{M},
\end{aligned}
$$

where we used (5.17) in the second inequality, taking $\varepsilon>0$ sufficiently small, and (5.15) in the third inequality. It remains to consider the case when the sum (5.32) is taken over $n_{1}=-n_{2}$ or $n_{1}=-n_{3}$. The contribution of the terms with $n_{1}=-n_{2} \neq-n_{3}$ is again handled exploiting again 
the map $n_{3} \leftrightarrow-n_{3}$, so that we have, for all $\varepsilon \in(0,1)$

$$
\begin{aligned}
& \text { (5.32) } \\
& \left.\right|_{n_{1}=-n_{2} \neq-n_{3}}=\frac{1}{2}\left|\sum_{\substack{\left|n_{1}\right|,\left|n_{3}\right| \leqslant N,\left|n_{1}\right| \neq\left|n_{3}\right| \\
\left|n_{1}\right|>M}} \frac{1}{n_{1}\left\langle n_{1}\right\rangle^{4 s}}\left(\frac{1}{\left(n_{3}-n_{1}\right)}-\frac{1}{\left(n_{3}+n_{1}\right)}\right)\right| \\
& =\frac{1}{2}\left|\sum_{\substack{\left|n_{1}\right|,\left|n_{3}\right| \leqslant N \\
\left|n_{1}\right| \neq\left|n_{3}\right| \\
\left|n_{1}\right|>M}} \frac{1}{\left\langle n_{1}\right\rangle^{4 s}} \frac{1}{\left(n_{3}^{2}-n_{1}^{2}\right)}\right| \lesssim \sum_{\substack{M<\left|n_{1}\right| \leqslant N \\
\left|n_{3}\right| \leqslant N, n_{3} \neq n_{1}}} \frac{1}{\left\langle n_{1}\right\rangle^{4 s}} \frac{1}{\left\langle n_{3}^{2}-n_{1}^{2}\right\rangle} \\
& \leqslant \sum_{M<\left|n_{1}\right| \leqslant N} \frac{C_{\varepsilon}}{\left\langle n_{1}\right\rangle^{4 s+1-\varepsilon}} \leqslant \frac{C_{\varepsilon}}{M^{4 s-\varepsilon}}
\end{aligned}
$$

where we used (5.17) in the last inequality.

By symmetry w.r.t. $n_{2} \leftrightarrow n_{3}$, using the triangle inequality, we can reduce to consider $n_{1}=-n_{2}$. We have

$$
\begin{aligned}
\left.(5.32)\right|_{n_{1}=n_{2}} & \lesssim \sum_{\substack{M<\left|n_{1}\right| \leqslant N \\
\left|n_{3}\right| \leqslant N}} \frac{1}{\left\langle n_{3}-n_{1}\right\rangle\left\langle n_{1}\right\rangle^{4 s+1}} \\
& \lesssim \sum_{\left|n_{3}\right| \leqslant N}\left(\sum_{M<\left|n_{1}\right| \leqslant N} \frac{1}{\left\langle n_{1}\right\rangle^{\frac{3}{2}(4 s+1)}}\right)^{2 / 3}\left(\sum_{M<\left|n_{1}\right| \leqslant N} \frac{1}{\left\langle n_{3}-n_{1}\right\rangle^{3}}\right)^{1 / 3}
\end{aligned}
$$

For the contribution of the terms with $n_{1}=-n_{2}=-n_{3}$, we have

$$
\left.(5.32)\right|_{n_{1}=-n_{2}=-n_{3}} \lesssim \sum_{M<\left|n_{1}\right| \leqslant N} \frac{1}{\left\langle n_{1}\right\rangle^{4 s+2}} \lesssim \frac{1}{M^{4 s+1}}
$$

- Case (e), $\sigma=(4,3,2,1)$. Note that $n_{1}+n_{2}=n_{\sigma(1)}+n_{\sigma(2)}$ and $n_{3}+n_{4}=n_{\sigma(3)}+n_{\sigma(4)}$ reduce to $n_{1}+n_{2}=n_{3}+n_{4}$, so we need to evaluate

$$
\begin{aligned}
& \left|\sum_{\substack{\left|n_{i}\right| \leqslant N, i=1, \ldots, 4 \\
n_{1} \neq n_{4}, n_{2} \neq n_{3} \\
n_{1}+n_{2}=n_{3}+n_{4}, \max _{i}\left(\left|n_{i}\right|\right)>M}} \frac{1}{\left(n_{4}-n_{1}\right)\left(n_{3}-n_{2}\right)\left\langle n_{1}\right\rangle^{2 s}\left\langle n_{2}\right\rangle^{2 s}}\right| \\
& \leqslant \sum_{\substack{\left|n_{i}\right| \leqslant N, i=1,2,3 \\
\max \left(\left|n_{1}\right|,\left|n_{2}\right|,\left|n_{3}\right|\right)>M / 3}} \frac{1}{\left\langle n_{2}-n_{3}\right\rangle^{2}\left\langle n_{1}\right\rangle^{2 s}\left\langle n_{2}\right\rangle^{2 s}},
\end{aligned}
$$

where we used that the sum was restricted over $n_{4}-n_{1}=n_{2}-n_{3}$. Since

$$
\sum_{\left|n_{3}\right| \leqslant N} \frac{1}{\left\langle n_{2}-n_{3}\right\rangle^{2}} \leqslant \sum_{\left|n_{3}\right|} \frac{1}{\left\langle n_{3}\right\rangle^{2}} \leqslant C,
$$

when $\left|n_{1}\right|>M / 3$ we can bound

$$
\left.(5.36)\right|_{\left|n_{1}\right|>M / 3} \leqslant \sum_{\substack{M / 3<\left|n_{1}\right| \leqslant N \\\left|n_{2}\right| \leqslant N}} \frac{1}{\left\langle n_{1}\right\rangle^{2 s}\left\langle n_{2}\right\rangle^{2 s}} \lesssim \sum_{M / 3<\left|n_{1}\right| \leqslant N} \frac{1}{\left\langle n_{1}\right\rangle^{2 s}} \lesssim \frac{1}{M^{2 s-1}}
$$

and when $\left|n_{2}\right|>M / 3$ we can bound

$$
\left.(5.36)\right|_{\left|n_{2}\right|>M / 3} \leqslant \sum_{\substack{M / 3<\left|n_{2}\right| \leqslant N \\\left|n_{1}\right| \leqslant N}} \frac{1}{\left\langle n_{1}\right\rangle^{2 s}\left\langle n_{2}\right\rangle^{2 s}} \lesssim \sum_{M / 3<\left|n_{2}\right| \leqslant N} \frac{1}{\left\langle n_{2}\right\rangle^{2 s}} \lesssim \frac{1}{M^{2 s-1}} .
$$


If $\left|n_{3}\right|>M / 3$ we use (5.14) to bound

$$
\begin{aligned}
\left.(5.36)\right|_{\left|n_{3}\right|>M / 3} & \leqslant \sum_{\substack{M / 3<\left|n_{3}\right| \leqslant N \\
\left|n_{1}\right| \leqslant N}} \frac{1}{\left\langle n_{1}\right\rangle^{2 s}} \sum_{\left|n_{2}\right| \leqslant N} \frac{1}{\left\langle n_{2}-n_{3}\right\rangle^{2}\left\langle n_{2}\right\rangle^{2 s}} \\
& \lesssim \sum_{\substack{M / 3<\left|n_{3}\right| \leqslant N \\
\left|n_{1}\right| \leqslant N}} \frac{1}{\left\langle n_{1}\right\rangle^{2 s}\left\langle n_{3}\right\rangle^{2 s}} \lesssim \sum_{M / 3<\left|n_{3}\right| \leqslant N} \frac{1}{\left\langle n_{3}\right\rangle^{2 s}} \lesssim \frac{1}{M^{2 s-1}} .
\end{aligned}
$$

To deal with the cases $(f),(g),(h)$ we will use the decomposition from Lemma 5.1 and the following elementary fact

$$
\left|n_{a}-n_{b}\right| \geqslant \min \left(\left|n_{a}\right|,\left|n_{b}\right|\right) \Rightarrow\left|n_{a}-n_{b}\right| \geqslant \frac{1}{2} \max \left(\left|n_{a}\right|,\left|n_{b}\right|\right) .
$$

By symmetry w.r.t. $a \leftrightarrow b$, this is a consequence of

$$
\left|n_{a}-n_{b}\right| \geqslant \min \left(\left|n_{a}\right|,\left|n_{b}\right|\right) \Rightarrow\left|n_{a}-n_{b}\right| \geqslant \frac{\left|n_{a}\right|}{2} .
$$

This is immediate if $\left|n_{b}\right| \geqslant \frac{\left|n_{a}\right|}{2}$, since then $\min \left(\left|n_{a}\right|,\left|n_{b}\right|\right) \geqslant \frac{\left|n_{a}\right|}{2}$. Otherwise one has $\left|n_{b}\right|<\frac{\left|n_{a}\right|}{2}$ and by triangle inequality

$$
\left|n_{a}-n_{b}\right| \geqslant\left|n_{a}\right|-\left|n_{b}\right|>\left|n_{a}\right|-\frac{\left|n_{a}\right|}{2}=\frac{\left|n_{a}\right|}{2} .
$$

- Case (f), $\sigma=(3,4,2,1)$. We decompose

$$
\left.\left|F_{N}-F_{M}\right|^{2}\right|_{\sigma=(3,4,2,1)} \leqslant\left. 2\left|F_{N}^{<}-F_{M}^{<}\right|^{2}\right|_{\sigma=(3,4,2,1)}+\left.2\left|F_{N}^{\geqslant}-F_{M} \geqslant\right|^{2}\right|_{\sigma=(3,4,2,1)}
$$

and we will bound these term separately. We will write $(5.40)^{<}$and $(5.40) \geqslant$ to denote the first and second term of the sum. Noting that $n_{1}+n_{2}=n_{\sigma(1)}+n_{\sigma(2)}$ and $n_{3}+n_{4}=n_{\sigma(3)}+n_{\sigma(4)}$ reduce to $n_{1}+n_{2}=n_{3}+n_{4}$, we get

$$
(5.40) \geqslant=2 \mid \sum_{\substack{\left|n_{i}\right| \leqslant N, i=1, \ldots, 4 \\ 0 \neq\left|n_{1}-n_{3}\right| \geqslant \min \left(\left|n_{1}\right|,\left|n_{3}\right|\right) \\ 0 \neq\left|n_{2}-n_{3}\right| \geqslant \min \left(\left|n_{2}\right|,\left|n_{3}\right|\right) \\ n_{1}+n_{2}=n_{3}+n_{4}, \max _{i}\left(\left|n_{i}\right|\right)>M}} \frac{\left|n_{3}\right|^{2 s}}{\left(n_{3}-n_{1}\right)\left(n_{3}-n_{2}\right)\left\langle n_{1}\right\rangle^{2 s}\left\langle n_{2}\right\rangle^{2 s}\left\langle n_{4}\right\rangle^{2 s} \mid}
$$

Since we are summing over

$$
\left|n_{1}-n_{3}\right| \geqslant \min \left(\left|n_{1}\right|,\left|n_{3}\right|\right), \quad\left|n_{2}-n_{3}\right| \geqslant \min \left(\left|n_{2}\right|,\left|n_{3}\right|\right),
$$

by (5.39) we can use

$$
\frac{1}{\left|n_{3}-n_{1}\right|}, \frac{1}{\left|n_{3}-n_{2}\right|} \lesssim \frac{1}{\left\langle n_{3}\right\rangle}
$$

Thus

$$
(5.40) \geqslant \lesssim \sum_{\substack{\left|n_{i}\right| \leqslant N, i=1,2,4 \\ \max \left(\left|n_{1}\right|,\left|n_{2}\right|,\left|n_{4}\right|\right)>M / 3}} \frac{1}{\left\langle n_{1}\right\rangle^{2 s}\left\langle n_{2}\right\rangle^{2 s}\left\langle n_{4}\right\rangle^{2 s}} \lesssim \frac{1}{M^{2 s-1}}
$$

To handle $(5.40)^{<}$we introduce

$$
\begin{aligned}
& A_{N, M}^{a, b}:=\left\{\left|n_{i}\right|,\left|m_{i}\right| \leqslant N, 0<\left|n_{a}-m_{a}\right|<\min \left(\left|n_{a}\right|,\left|m_{a}\right|\right),\right. \\
&\left.n_{a}+n_{b}=m_{a}+m_{b}, \max \left(\left|m_{a}\right|,\left|m_{b}\right|,\left|n_{a}\right|,\left|n_{b}\right|\right)>M\right\},
\end{aligned}
$$

so that Lemma 5.1 gives

$$
\left|F_{N}^{<}-F_{M}^{<}\right|=2\left|\operatorname{Re}\left(\sum_{k \geqslant 1} \frac{(s)_{k}}{k !} \sum_{A_{N, M}^{1,2}} \frac{\left(m_{1}-n_{1}\right)^{k-1}}{\left|n_{1}\right|^{k}}\left|m_{1}\right|^{s}\left|n_{1}\right|^{s} u\left(n_{1}\right) u\left(n_{2}\right) \bar{u}\left(m_{1}\right) \bar{u}\left(m_{2}\right)\right)\right| .
$$


and squaring

$$
\begin{aligned}
& \left|F_{N}^{<}(u)-F_{M}^{<}(u)\right|^{2} \\
& =4 \sum_{A_{N, M}^{1,2} \times A_{N, M}^{3,4}}\left[\sum_{k, h \geqslant 1} \frac{(s)_{k}(s)_{h}}{k ! h !} \frac{\left(m_{1}-n_{1}\right)^{k-1}\left(n_{3}-m_{3}\right)^{h-1}}{\left|n_{1}\right|^{k}\left|m_{3}\right|^{h}}\left|m_{1}\right|^{s}\left|n_{1}\right|^{s}\left|n_{3}\right|^{s}\left|m_{3}\right|^{s}\right] \prod_{j=1}^{4} \bar{u}\left(m_{j}\right) u\left(n_{j}\right) .
\end{aligned}
$$

We now taking the expected value w.r.t. $\gamma_{s}$, using again formula (5.13) with $\ell=4$, and we restrict the sum to the contribution of a single permuation $\sigma$, so that we get

$$
\begin{aligned}
& \left.\left\|F_{N}^{<}-F_{M}^{<}\right\|_{L^{2}\left(\gamma_{s}\right)}^{2}\right|_{\sigma} \\
& =4 \sum_{A_{N, M}^{1,2} \times A_{N, M}^{3,4}}\left[\sum_{k, h \geqslant 1} \frac{(s)_{k}(s)_{h}}{k ! h !} \frac{\left(m_{1}-n_{1}\right)^{k-1}\left(n_{3}-m_{3}\right)^{h-1}}{\left|n_{1}\right|^{k}\left|m_{3}\right|^{h}}\left|m_{1}\right|^{s}\left|n_{1}\right|^{s}\left|n_{3}\right|^{s}\left|m_{3}\right|^{s}\right] \prod_{j=1}^{4} \frac{\delta_{m_{j}, n_{\sigma(j)}}}{\left\langle n_{j}\right\rangle^{2 s}} \\
& =4 \sum_{\sigma\left(A_{N, M}^{1,2} \times A_{N, M}^{3,4}\right)}\left[\sum_{k, h \geqslant 1} \frac{(s)_{k}(s)_{h}}{k ! h !} \frac{\left(n_{\sigma(1)}-n_{1}\right)^{k-1}\left(n_{3}-n_{\sigma(3)}\right)^{h-1}}{\left|n_{1}\right|^{k}\left|n_{\sigma(3)}\right|^{h}}\left|n_{\sigma(1)}\right|^{s}\left|n_{1}\right|^{s}\left|n_{3}\right|^{s}\left|n_{\sigma(3)}\right|^{s}\right] \prod_{j=1}^{4} \frac{1}{\left\langle n_{j}\right\rangle^{2 s}} \\
& \lesssim \sum_{\sigma\left(A_{N, M}^{1,2} \times A_{N, M}^{3,4}\right)} \frac{\left|n_{1}\right|^{s-1}\left|n_{\sigma(3)}\right|^{s-1}\left|n_{\sigma(1)}\right|^{s}\left|n_{3}\right|^{s}}{\prod_{j=1}^{4}\left\langle n_{j}\right\rangle^{2 s}}
\end{aligned}
$$

So that

which implies

$$
\left.(5.40)^{<} \lesssim \sum_{\sigma\left(A_{N, M}^{1,2} \times A_{N, M}^{3,4}\right)} \frac{\left|n_{1}\right|^{s-1}\left|n_{\sigma(3)}\right|^{s-1}\left|n_{\sigma(1)}\right|^{s}\left|n_{3}\right|^{s}}{\prod_{j=1}^{4}\left\langle n_{j}\right\rangle^{2 s}}\right|_{\sigma=(3,4,2,1)}
$$

$$
\begin{aligned}
& (5.40)^{<} \lesssim \sum_{\substack{\left|n_{i}\right| \leqslant N, i=1, \ldots 4 \\
n_{1}+n_{2}=n_{3}+n_{4} \\
0<\left|n_{2}-n_{3}\right|<\min \left(\left|n_{2}\right|,\left|n_{3}\right|\right) \\
0<\left|n_{1}-n_{3}\right|<\min \left(\left|n_{1}\right|,\left|n_{3}\right|\right) \\
\max _{i}\left(\left|n_{i}\right|\right)>M}} \frac{1}{\left\langle n_{1}\right\rangle^{s+1}\left\langle n_{2}\right\rangle^{s+1}\left\langle n_{4}\right\rangle^{2 s}} \\
& \lesssim \sum_{\substack{\left|n_{1}\right|,\left|n_{2}\right|,\left|n_{4}\right| \leqslant N \\
\max \left(\left|n_{1}\right|,\left|n_{2}\right|,\left|n_{4}\right|\right) \geqslant M / 3}} \frac{1}{\left\langle n_{1}\right\rangle^{s+1}\left\langle n_{2}\right\rangle^{s+1}\left\langle n_{4}\right\rangle^{2 s}} \lesssim \frac{1}{M^{2 s-1}} .
\end{aligned}
$$

- Case $(\mathrm{g}), \sigma=(3,4,1,2)$. Proceeding as in the case (f), we decompose

$$
\left.\left|F_{N}-F_{M}\right|^{2}\right|_{\sigma=(3,4,2,1)} \leqslant\left. 2\left|F_{N}^{<}-F_{M}^{<}\right|^{2}\right|_{\sigma=(3,4,2,1)}+\left.2\left|F_{N}^{\geqslant}-F_{M} \geqslant\right|^{2}\right|_{\sigma=(3,4,2,1)}
$$

and we will bound these term separately. We will write $(5.42)^{<}$and $(5.42) \geqslant$ to denote the first and second term of the sum. Note that $n_{1}+n_{2}=n_{\sigma(1)}+n_{\sigma(2)}$ and $n_{3}+n_{4}=n_{\sigma(3)}+n_{\sigma(4)}$ reduce to $n_{1}+n_{2}=n_{3}+n_{4}$, so

$$
(5.42) \geqslant=2\left|\sum_{\substack{\left|n_{i}\right| \leqslant N, i=1, \ldots, 4 \\ 0 \neq\left|n_{1}-n_{3}\right| \geqslant \min \left(\left|n_{1}\right|,\left|n_{3}\right|\right) \\ n_{1}+n_{2}=n_{3}+n_{4}, \max _{i}\left(\left|n_{i}\right|\right)>M}} \frac{\left|n_{3}\right|^{2 s}}{\left(n_{3}-n_{1}\right)^{2}\left\langle n_{1}\right\rangle^{2 s}\left\langle n_{2}\right\rangle^{2 s}\left\langle n_{4}\right\rangle^{2 s}}\right|
$$

by (5.39) we can use

so that

$$
\frac{1}{\left|n_{3}-n_{1}\right|} \lesssim \frac{1}{\left\langle n_{3}\right\rangle}
$$

$$
(5.42) \geqslant \lesssim \sum_{\substack{\left|n_{i}\right| \leqslant N, i=1,2,4 \\ \max \left(\left|n_{1}\right|,\left|n_{2}\right|,\left|n_{4}\right|\right)>M / 3}} \frac{1}{\left\langle n_{1}\right\rangle^{2 s}\left\langle n_{2}\right\rangle^{2 s}\left\langle n_{4}\right\rangle^{2 s}} \lesssim \frac{1}{M^{2 s-1}}
$$


To handle $(5.42)^{<}$we use again (see case (f))

$$
\left.(5.42)^{<} \lesssim \sum_{\sigma\left(A_{N, M}^{1,2} \times A_{N, M}^{3,4}\right)} \frac{\left|n_{1}\right|^{s-1}\left|n_{\sigma(3)}\right|^{s-1}\left|n_{\sigma(1)}\right|^{s}\left|n_{3}\right|^{s}}{\prod_{j=1}^{4}\left\langle n_{j}\right\rangle^{2 s}}\right|_{\sigma=(3,4,1,2)},
$$

which implies

$$
\begin{aligned}
& (5.40)^{<} \lesssim \sum_{\substack{\left.\left|n_{i}\right| \leqslant N, i=1, \ldots 4 \\
n_{1}+n_{2}=n_{3}+n_{4} \\
0<\left|n_{1}-n_{3}\right|<\min \left(\left|n_{1}\right|,\left|n_{3}\right|\right) \\
\max _{i}\left(\left|n_{i}\right|\right)\right\rangle M}} \frac{1}{\left\langle n_{1}\right\rangle^{2}\left\langle n_{2}\right\rangle^{2 s}\left\langle n_{4}\right\rangle^{2 s}} \\
& \lesssim \sum_{\substack{\left|n_{1}\right|,\left|n_{2}\right|,\left|n_{4}\right| \leqslant N \\
\max \left(\left|n_{1}\right|,\left|n_{2}\right|,\left|n_{4}\right|\right) \geqslant M / 3}} \frac{1}{\left\langle n_{1}\right\rangle^{2}\left\langle n_{2}\right\rangle^{2 s}\left\langle n_{4}\right\rangle^{2 s}} \lesssim \frac{1}{M^{2 s-1}} .
\end{aligned}
$$

- Case (h), $\sigma=(4,3,1,2)$. Proceeding as in the case (f), we decompose

$$
\left.\left|F_{N}-F_{M}\right|^{2}\right|_{\sigma=(4,3,1,2)} \leqslant\left. 2\left|F_{N}^{<}-F_{M}^{<}\right|^{2}\right|_{\sigma=(4,3,1,2)}+\left.2\left|F_{N}^{\geqslant}-F_{M}^{\geqslant}\right|^{2}\right|_{\sigma=(4,3,1,2)}
$$

and we will bound these term separately. We will write $(5.45)^{<}$and $(5.45) \geqslant$ to denote the first and second term of the sum.

Note that $n_{1}+n_{2}=n_{\sigma(1)}+n_{\sigma(2)}$ and $n_{3}+n_{4}=n_{\sigma(3)}+n_{\sigma(4)}$ reduce to $n_{1}+n_{2}=n_{3}+n_{4}$, so we need to evaluate

$$
\begin{aligned}
& (5.45) \geqslant \lesssim\left|\sum_{\substack{\left.\left|n_{i}\right| \leqslant N, i=1, \ldots, 4 \\
0 \neq\left|n_{1}-n_{4}\right| \geqslant \min \left(\left|n_{1}\right|,\left|n_{4}\right|\right) \\
0 \neq\left|n_{1}-n_{3}\right| \geqslant \min \left(n_{1}|,| n_{3} \mid\right) \\
n_{1}+n_{2}=n_{3}+n_{4}, \max _{i}\left(\left|n_{i}\right|\right)\right\rangle M}} \frac{1}{\left(n_{4}-n_{1}\right)\left(n_{3}-n_{1}\right)\left\langle n_{1}\right\rangle^{2 s}\left\langle n_{2}\right\rangle^{2 s}}\right| \\
& \lesssim \sum_{\substack{\left.\left|n_{i}\right| \leqslant N, i=1, \ldots, 4 \\
\max \left(\left|n_{1}\right|,\left|n_{2}\right|,\left|n_{4}\right|\right)\right\rangle M / 3}} \frac{1}{\left\langle n_{4}\right\rangle\left\langle n_{2}-n_{4}\right\rangle\left\langle n_{1}\right\rangle^{2 s}\left\langle n_{2}\right\rangle^{2 s}}
\end{aligned}
$$

where we used (5.39), so that

$$
\frac{1}{\left|n_{4}-n_{1}\right|} \lesssim \frac{1}{\left\langle n_{4}\right\rangle}
$$

and the fact that the sum were restricted to $n_{3}-n_{1}=n_{2}-n_{4}$ If $\left|n_{1}\right|>M / 3$ we can estimate

$$
\begin{aligned}
(5.45) \geqslant\left.\right|_{\left|n_{1}\right|>M / 3} & \lesssim \sum_{\substack{M / 3<\left|n_{1}\right| \leqslant N,\left|n_{4}\right| \leqslant N}} \frac{1}{\left\langle n_{1}\right\rangle^{2 s}\left\langle n_{4}\right\rangle} \sum_{\left|n_{2}\right| \leqslant N} \frac{1}{\left\langle n_{2}\right\rangle^{2 s}\left\langle n_{2}-n_{4}\right\rangle} \\
& \lesssim \sum_{\substack{M / 3<\left|n_{1}\right| \leqslant N \\
\left|n_{4}\right| \leqslant N}} \frac{1}{\left\langle n_{1}\right\rangle^{2 s}\left\langle n_{4}\right\rangle^{2}} \lesssim \sum_{M / 3<\left|n_{1}\right| \leqslant N} \frac{1}{\left\langle n_{1}\right\rangle^{2 s}} \lesssim \frac{1}{M^{2 s-1}},
\end{aligned}
$$

where in the second inequality we used (5.14). If $\left|n_{4}\right|>M / 3$ we estimate similarly

$$
\begin{aligned}
(5.45) \geqslant\left.\right|_{\left|n_{4}\right|>M / 3} & \lesssim \sum_{\substack{M / 3<\left|n_{4}\right| \leqslant N,\left|n_{1}\right| \leqslant N}} \frac{1}{\left\langle n_{1}\right\rangle^{2 s}\left\langle n_{4}\right\rangle} \sum_{\left|n_{2}\right| \leqslant N} \frac{1}{\left\langle n_{2}\right\rangle^{2 s}\left\langle n_{2}-n_{4}\right\rangle} \\
& \lesssim \sum_{\substack{M / 3<\left|n_{4}\right| \leqslant N,\left|n_{1}\right| \leqslant N}} \frac{1}{\left\langle n_{1}\right\rangle^{2 s}\left\langle n_{4}\right\rangle^{2}} \lesssim \sum_{M / 3<\left|n_{4}\right| \leqslant N} \frac{1}{\left\langle n_{4}\right\rangle^{2}} \lesssim \frac{1}{M} .
\end{aligned}
$$


If $\left|n_{2}\right|>M / 3$ we use that by Cauchy-Schwartz

$$
\begin{aligned}
\sum_{\left|n_{4}\right| \leqslant N} \frac{1}{\left\langle n_{4}\right\rangle\left\langle n_{2}-n_{4}\right\rangle} & \leqslant\left(\sum_{\left|n_{4}\right| \leqslant N} \frac{1}{\left\langle n_{4}\right\rangle^{2}}\right)^{1 / 2}\left(\sum_{\left|n_{4}\right| \leqslant N} \frac{1}{\left\langle n_{2}-n_{4}\right\rangle^{2}}\right)^{1 / 2} \\
& \leqslant\left(\sum_{\left|n_{4}\right|} \frac{1}{\left\langle n_{4}\right\rangle^{2}}\right)^{2} \leqslant C
\end{aligned}
$$

so that

$$
\begin{aligned}
(5.45) \geqslant\left.\right|_{\left|n_{2}\right|>M / 3} & \lesssim \sum_{\substack{M / 3<\left|n_{2}\right| \leqslant N \\
\left|n_{1}\right| \leqslant N}} \frac{1}{\left\langle n_{1}\right\rangle^{2 s}\left\langle n_{2}\right\rangle^{2 s}} \sum_{\left|n_{4}\right| \leqslant N} \frac{1}{\left\langle n_{4}\right\rangle\left\langle n_{2}-n_{4}\right\rangle} \\
& \lesssim \sum_{\substack{M / 3<\left|n_{2}\right| \leqslant N,\left|n_{1}\right| \leqslant N}} \frac{1}{\left\langle n_{1}\right\rangle^{2 s}\left\langle n_{2}\right\rangle^{2 s}} \lesssim \sum_{\substack{M / 3<\left|n_{2}\right| \leqslant N,\left|n_{1}\right| \leqslant N}} \frac{1}{\left\langle n_{2}\right\rangle^{2 s}} \lesssim \frac{1}{M^{2 s-1}},
\end{aligned}
$$

To handle $(5.42)^{<}$we use again (see case $\left.(\mathrm{f})\right)$

$$
\left.(5.45)^{<} \lesssim \sum_{\sigma\left(A_{N, M}^{1,2} \times A_{N, M}^{3,4}\right)} \frac{\left|n_{1}\right|^{s-1}\left|n_{\sigma(3)}\right|^{s-1}\left|n_{\sigma(1)}\right|^{s}\left|n_{3}\right|^{s}}{\prod_{j=1}^{4}\left\langle n_{j}\right\rangle^{2 s}}\right|_{\sigma=(4,3,1,2)},
$$

which implies

$$
(5.45)^{<} \lesssim \sum_{\substack{\left|n_{i}\right| \leqslant N, i=1, \ldots 4 \\ n_{1}+n_{2}=n_{3}+n_{4} \\ 0<\left|n_{1}-n_{3}\right|<\min \left(\left|n_{1}\right|,\left|n_{3}\right|\right) \\ 0<\left|n_{1}-n_{4}\right|<\min \left(\left|n_{1}\right|,\left|n_{4}\right|\right) \\ \max _{i}\left(\left|n_{i}\right|\right)>M}} \frac{1}{\left\langle n_{1}\right\rangle^{2}\left\langle n_{2}\right\rangle^{2 s}\left\langle n_{3}\right\rangle^{s}\left\langle n_{4}\right\rangle^{s}}
$$

To handle this we use the following elementary fact

$$
\left|n_{a}-n_{b}\right|<\min \left(\left|n_{a}\right|,\left|n_{b}\right|\right) \Rightarrow\left|n_{a}\right| \simeq\left|n_{b}\right|,
$$

By symmetry w.r.t. $n_{a} \leftrightarrow n_{b}$ it suffices to show

$$
\left|n_{a}\right| \leqslant 2\left|n_{b}\right|
$$

which follows by triangle inequality

$$
\left|n_{a}\right| \leqslant\left|n_{b}\right|+\left|n_{a}-n_{b}\right| \leqslant 2\left|n_{b}\right|,
$$

where we used the assumption in (5.49) in the second inequality. Using (5.49) we see that the sum above is in fact restricted to

$$
\left|n_{1}\right| \simeq\left|n_{3}\right| \simeq\left|n_{4}\right|
$$

which with the restrictions

$$
n_{1}+n_{2}=n_{3}+n_{4}, \quad \max _{i}\left(\left|n_{i}\right|\right)>M
$$

also forces

$$
\left|n_{1}\right| \simeq\left|n_{3}\right| \simeq\left|n_{4}\right| \gtrsim M
$$

Thus we can estimate

$$
(5.45)^{<} \lesssim \frac{1}{M^{s}} \sum_{\substack{\left|n_{1}\right|,\left|n_{2}\right|,\left|n_{3}\right| \leqslant N \\\left|n_{1}\right|,\left|n_{3}\right|>M}} \frac{1}{\left\langle n_{1}\right\rangle^{1+\frac{s}{2}}\left\langle n_{2}\right\rangle^{2 s}\left\langle n_{3}\right\rangle^{1+\frac{s}{2}}} \lesssim \frac{1}{M^{\frac{3}{2} s}},
$$

that concludes the proof. 
Let us recall once more

$$
F_{N}:=\left.\frac{d}{d \alpha}\left\|\mathscr{G}_{\alpha} P_{N} u\right\|_{\dot{H}^{s}}\right|_{\alpha=0} .
$$

By Lemma 5.5 there is $C>0$ such that for any $M \geqslant N \in \mathbb{N}$

$$
\left\|F_{N}-F_{M}\right\|_{L^{2}\left(\gamma_{s}\right)} \leqslant \frac{C}{N^{s^{*}}}, \quad s^{*}:=\min \left(\frac{1}{2}, s-\frac{1}{2}\right) .
$$

Note $s^{*}>0$ for $s>\frac{1}{2}$. Recalling that $F_{N}$ can be written as in (5.12), we immediately see that (6.1) implies by hypercontractivity that for all $p>2$ there is $C>0$ (possibly different from above) for which

$$
\left\|F_{N}-F_{M}\right\|_{L^{p}\left(\gamma_{s}\right)} \leqslant \frac{C p^{2}}{N^{s^{*}}}
$$

Then we can immediately establish the following concentration inequality for $F_{N}$ around its limit.

Proposition 6.1. Let $s>1 / 2$ and $N \in \mathbb{N}$. There are $C, c>0$ such that

$$
\gamma_{s}\left(\left|F_{N}-F\right| \geqslant t\right) \leqslant C e^{-c \sqrt{t} N^{\frac{s^{*}}{2}}}
$$

Proof. Having bounded all the moments as in (6.2) we can bound also the fractional exponential moment

$$
E_{s}\left[\exp \left(c \sqrt{\left|F_{N}-F_{M}\right|} N^{s^{*}}\right)\right]<\infty
$$

for a suitable constant $c>0$ (see e.g. [32, Proposition 4.5]). From (6.4) we obtain (6.3) in the standard way using Markov inequality.

These bounds (notably independent on $R$ ) are however not optimal and we need to improve on them. We shall show that $\left\{F_{N}\right\}_{N \in \mathbb{N}}$ are in fact sub-exponential random variables uniformly in $N$, whereby Proposition 3.1 will follow as a simple corollary. We split the bulk and the tail of the distribution of $F$ as follows:

Proposition 6.2. Let $s>1 / 2$ and $R^{*}:=\max \left(R^{\frac{2}{2 s-1}}, R^{2(2 s-1)}\right)$. Then There exist $c, C>0$ independent on $N$ for which

$$
\tilde{\gamma}_{s, N}\left(\left|F_{N}\right| \geqslant t\right)<C \exp \left(-\frac{c t}{R^{*}}\right), \quad t \leqslant N^{s^{*}} .
$$

Proposition 6.3. Let $s>1 / 2$ and $R^{*}:=\max \left(R^{\frac{2}{2 s-1}}, R^{2(2 s-1)}\right)$. There exist $c, C>0$ independent on $N$ for which

$$
\tilde{\gamma}_{s, N}\left(\left|F_{N}\right| \geqslant t\right)<C \exp \left(-\frac{c t}{R^{*}}\right), \quad t \geqslant N^{s^{*}} .
$$

To bound the tail of the distribution of $F$ we need some work and therefore that will be addressed first. Recall that for $j \in \mathbb{N}$ the Littlewood-Paley projector is denoted by $\Delta_{j}:=P_{2^{j}}-P_{2^{j-1}}$; we write $|n| \simeq 2^{j}$ to shorten $2^{j-1} \leqslant|n| \leqslant 2^{j}$ for $j \in \mathbb{N}$, while for $j=0|n| \simeq 1$ shortens $|n| \leqslant 1$.

Let us now shorten

$$
\begin{aligned}
X_{j, N} & :=2^{j\left(s-\frac{1}{2}\right)}\left\|\Delta_{j} P_{N} u\right\|_{L^{2}}, \quad X_{N}:=\sum_{j \geqslant 0} X_{j, N} \\
Y_{j, N} & :=\sum_{|n| \simeq 2^{j}}\left|\left(P_{N} u\right)(n)\right|, \quad Y_{N}:=\sum_{j \geqslant 0} Y_{j, N} .
\end{aligned}
$$

Then we obtain the following crucial lemma, whose proof can be found at the end of this section.

Lemma 6.4. We have

$$
\left|F_{N}\right| \lesssim X_{N}^{2} Y_{N}^{2}
$$


Lemma 6.4 allows us to bound

$$
\tilde{\gamma}_{s, N}\left(\left|F_{N}\right| \geqslant t\right) \leqslant \tilde{\gamma}_{s, N}\left(X_{N} Y_{N} \geqslant \sqrt{t}\right) \leqslant \tilde{\gamma}_{s, N}\left(X_{N} \geqslant t^{\frac{1}{2}-\frac{1}{4 s}}\right)+\tilde{\gamma}_{s, N}\left(Y_{N} \geqslant t^{\frac{1}{4 s}}\right),
$$

so that we can treat the two contributes separately.

Lemma 6.5. There are $C, c>0$ and $l>1$ such that

$$
\tilde{\gamma}_{s, N}\left(X_{N} \geqslant t^{\frac{1}{2}-\frac{1}{4 s}}\right) \leqslant \exp \left(-\frac{c t}{R^{\frac{2}{2 s-1}}}\right), \quad \forall t \geqslant\left(\log _{2} N\right)^{\frac{4 s}{2 s-1} l} .
$$

Proof. Let

and split

$$
j_{t}:=\left\lfloor\log _{2} \frac{t^{\frac{1}{2 s}}}{R^{\frac{2}{2 s-1}}}\right\rfloor
$$

$$
X_{N}=\sum_{0 \leqslant j \leqslant j_{t}} X_{j, N}+\sum_{j>j_{t}} X_{j, N}
$$

Since by definition of $\tilde{\gamma}_{s, N}$ we have $\left\|P_{N} u\right\|_{L^{2}} \leqslant R$ on a set of full $\tilde{\gamma}_{s, N}$ measure, the following bound

$$
\sum_{0 \leqslant j \leqslant j_{t}} X_{j, N} \leqslant \sum_{0 \leqslant j \leqslant j_{t}} 2^{j\left(s-\frac{1}{2}\right)}\left\|\Delta_{j} P_{N} u\right\|_{L^{2}} \leqslant R 2^{j_{t}\left(s-\frac{1}{2}\right)} \leqslant t^{\frac{1}{2}-\frac{1}{4 s}},
$$

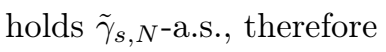

$$
\tilde{\gamma}_{s, N}\left(\sum_{0 \leqslant j \leqslant j_{t}} X_{j, N} \geqslant t^{\frac{1}{2}-\frac{1}{4 s}}\right)=0 .
$$

Now we analyse the second summand of (6.12). Let $c_{0}>0$ small enough so that

$$
\sigma_{j}:=c_{0} j^{-l}, \quad \sum_{j \in \mathbb{N}} \sigma_{j} \leqslant 1
$$

For any $j \in \mathbb{N}$ we have

$$
\tilde{\gamma}_{s, N}\left(X_{j, N} \geqslant \sigma_{j} t^{\frac{1}{2}-\frac{1}{4 s}}\right)=\tilde{\gamma}_{s, N}\left(2^{j\left(s-\frac{1}{2}\right)}\left\|\Delta_{j} P_{N} u\right\|_{L^{2}} \geqslant \sigma_{j} t^{\frac{1}{2}-\frac{1}{4 s}}\right) .
$$

By Bernstein inequality (1.14)

$$
\begin{aligned}
\tilde{\gamma}_{s, N}\left(2^{j\left(s-\frac{1}{2}\right)}\left\|\Delta_{j} P_{N} u\right\|_{L^{2}} \geqslant \sigma_{j} t^{\frac{1}{2}-\frac{1}{4 s}}\right) & =\tilde{\gamma}_{s, N}\left(\left\|\Delta_{j} P_{N} u\right\|_{L^{2}}^{2} \geqslant 2^{-2 j\left(s-\frac{1}{2}\right)} \sigma_{j}^{2} t^{2 \frac{2 s-1}{4 s}}\right) \\
& \leqslant C \exp \left(-c 2^{j} \min \left(\sigma_{j}^{2} t^{\frac{2 s-1}{2 s}}, \sigma_{j}^{4} t^{\frac{2 s-1}{s}}\right)\right) .
\end{aligned}
$$

Therefore for any fixed $j$ we have

$$
\tilde{\gamma}_{s, N}\left(2^{j\left(s-\frac{1}{2}\right)}\left\|\Delta_{j} P_{N} u\right\|_{L^{2}} \geqslant \sigma_{j} t^{\frac{1}{2}-\frac{1}{4 s}}\right) \leqslant C e^{-\sigma_{j}^{2} t^{\frac{2 s-1}{2 s}} 2^{-j}}
$$

provided

$$
t \gtrsim j^{\frac{4 s}{2 s-1} l} .
$$

Therefore the estimate extends to all $j \leqslant\left\lceil\log _{2} N\right\rceil$ for

$$
t \gtrsim\left(\log _{2} N\right)^{\frac{4 s}{2 s-1} l} .
$$

Then

$$
\begin{aligned}
\tilde{\gamma}_{s, N}\left(\sum_{j>j_{t}} X_{j, N} \geqslant t^{\frac{1}{2}-\frac{1}{4 s}}\right) & \leqslant \sum_{j>j_{t}} \tilde{\gamma}_{s, N}\left(X_{j, N} \geqslant \sigma_{j} t^{\frac{1}{2}-\frac{1}{4 s}}\right) \\
& \lesssim \sum_{j>j_{t}} e^{-2^{j} \sigma_{j}^{2} t^{\frac{2 s-1}{2 s}}} \leqslant C \exp \left(-c \frac{t}{R^{\frac{2}{2 s-1}}}\right)
\end{aligned}
$$

for some absolute constants $C, c>0$. 
Lemma 6.6. There is $c>0$ such that

$$
\tilde{\gamma}_{s, N}\left(Y_{N} \geqslant t^{\frac{1}{4 s}}\right) \leqslant \exp \left(-\frac{c t}{R^{2(2 s-1)}}\right) .
$$

Proof. We set now

$$
j_{t}:=\left\lfloor\log _{2} \frac{t^{\frac{1}{2 s}}}{R^{2}}\right\rfloor .
$$

Since $\left\|P_{N} u\right\|_{L^{2}} \leqslant R$ on a set of full $\tilde{\gamma}_{s, N}$ measure, the simple inequality

$$
Y_{j, N} \leqslant 2^{\frac{j}{2}}\left\|\Delta_{j} P_{N}\right\|_{L^{2}}
$$

implies the following bound to hold $\tilde{\gamma}_{s, N}$-a.s.

$$
\sum_{0 \leqslant j \leqslant j_{t}} Y_{j, N} \leqslant \sum_{0 \leqslant j \leqslant j_{t}} 2^{\frac{j}{2}}\left\|\Delta_{j} P_{N} u\right\|_{L^{2}} \leqslant R 2^{\frac{j_{t}}{2}}=t^{\frac{1}{4 s}} .
$$

Thus

$$
\tilde{\gamma}_{s, N}\left(\sum_{0 \leqslant j \leqslant j_{t}} Y_{j, N} \geqslant t^{\frac{1}{4 s}}\right)=0 .
$$

To estimate the contribution for $j>j_{t}$ we consider again the $\sigma_{j}>0$ defined in (6.14) and bound

$$
\tilde{\gamma}_{s, N}\left(\sum_{j>j_{t}} Y_{j, N} \geqslant t^{\frac{1}{4 s}}\right) \leqslant \sum_{j>j_{t}} \tilde{\gamma}_{s, N}\left(Y_{j, N} \geqslant \sigma_{j} t^{\frac{1}{4 s}}\right) .
$$

We have the following estimate for $Y_{j, N}$ from Hoeffding inequality

$$
\tilde{\gamma}_{s, N}\left(Y_{j, N} \geqslant t\right) \leqslant C \exp \left(-\frac{c t^{2}}{\sum_{|n| \simeq 2^{j}}\langle n\rangle^{-2 s}}\right)
$$

for suitable constants $C, c>0$. Therefore

$$
\tilde{\gamma}_{s, N}\left(Y_{j, N} \geqslant \sigma_{j} t^{\frac{1}{4 s}}\right) \lesssim e^{-c \sigma_{j}^{2} 2^{j(2 s-1)} t^{\frac{1}{2 s}}} \quad \text { for some } c>0 .
$$

Thus, noting

there is some $C, c>0$ such that we can bound

$$
2^{j_{t}(2 s-1)} t^{\frac{1}{2 s}}=\frac{t}{R^{2(2 s-1)}}
$$

$$
\text { r.h.s. of }(6.19) \lesssim \sum_{j>j_{t}} e^{-c \sigma_{j}^{2} 2^{j(2 s-1)} t^{\frac{1}{2 s}}} \leqslant C \exp \left(-\frac{c t}{R^{2(2 s-1)}}\right),
$$

that concludes the proof.

Combining (6.10) with Lemma 6.5 and Lemma 6.6 we prove Proposition 6.3. Finally the bulk of the distribution of $F$ is easier to bound.

Proof of Proposition 6.2. Let us set $T:=\left\lfloor t^{\frac{1}{s^{*}}}\right\rfloor$. Notice that since we restrict to $t \leqslant N^{s^{*}}$ we have $N>T$. We use the union bound

$$
\tilde{\gamma}_{s, N}\left(\left|F_{N}\right| \geqslant t\right) \leqslant \gamma_{s}\left(\left|F_{N}-F_{T}\right| \geqslant t / 2\right)+\tilde{\gamma}_{s, N}\left(\left|F_{T}\right| \geqslant t / 2\right) .
$$

By Proposition 6.1 we have

$$
\gamma_{s}\left(\left|F_{N}-F_{T}\right| \geqslant t\right) \leqslant C e^{-c \sqrt{t} T^{\frac{s^{*}}{2}}} \leqslant C e^{-c t} .
$$

On the other hand since $t>T^{s^{*}}$ the estimate (6.6) applies to the second summand of (6.23). This concludes the proof. 
Proof of Lemma 6.4. The first step is to revisit the decomposition of Lemma 5.1. We set

$$
\begin{aligned}
F_{N}^{\geqslant} & :=\sum_{\substack{j_{1}, \ell_{1} \in \mathbb{N} \\
J \geqslant \min \left(j_{1}, \ell_{1}\right)}} \operatorname{Im} \int\left(\Delta_{j_{1}} P_{N} \bar{u}\right)^{(2 s)}\left(\Delta_{\ell_{1}} P_{N} u\right)\left(\Delta_{J} \mathcal{I}\left[P_{N} u\right]\right), \\
F_{N}^{<} & :=\sum_{\substack{j_{1}, \ell_{1} \in \mathbb{N} \\
J<\min \left(j_{1}, \ell_{1}\right)}} \operatorname{Im} \int\left(\Delta_{j_{1}} P_{N} \bar{u}\right)^{(2 s)}\left(\Delta_{\ell_{1}} P_{N} u\right)\left(\Delta_{J} \mathcal{I}\left[P_{N} u\right]\right) .
\end{aligned}
$$

Of course it is

$$
F_{N}=F_{N} \geqslant F_{N}^{<}
$$

With a slight abuse of notation we denote these two quantities by the same symbols as the ones of the decomposition of Lemma 5.1. This will lighten the exposition and anyway the analogy between the two representations is clear. We will also need

$$
\Delta_{J} \mathcal{I}\left[P_{N} u\right]=i \sum_{j_{2}, \ell_{1} \in \mathbb{N}} \sum_{\substack{\left|n_{2}\right|,\left|m_{2}\right| \leqslant N \\\left|n_{2}\right| \simeq 2^{j_{2}},\left|m_{2}\right| \simeq 2^{\ell_{2}} \\ 0 \neq\left|n_{2}-m_{2}\right| \simeq 2^{J}}} e^{i\left(n_{2}-m_{2}\right) x} \frac{\bar{u}\left(m_{2}\right) u\left(n_{2}\right)}{n_{2}-m_{2}}
$$

Using (6.28) we bound

$$
\left|F_{N} \geqslant\right| \lesssim \sum_{\substack{j_{1}, j_{2}, \ell_{1}, \ell_{2} \in \mathbb{N} \\ J \geqslant \min \left(\ell_{1}, j_{1}\right)}} \sum_{\substack{\left|n_{1}\right|,\left|n_{2}\right|,\left|m_{1}\right|,\left|m_{2}\right| \leqslant N \\\left|m_{1}\right| \simeq 2^{\ell_{1}},\left|n_{1}\right| \simeq 2^{j_{1}} \\\left|n_{2}\right| \simeq 2^{j_{2}},\left|m_{2}\right| \simeq 2^{\ell_{2}} \\ 0 \neq\left|n_{1}-m_{1}\right| \simeq 2^{J} \\ n_{1}+n_{2}=m_{1}+m_{2}}} \frac{\left|m_{1}\right|^{2 s}}{m_{1}-n_{1}} \bar{u}\left(m_{1}\right) \bar{u}\left(m_{2}\right) u\left(n_{1}\right) u\left(n_{2}\right) \mid .
$$

The constraints

$$
\left|n_{1}-m_{1}\right| \simeq 2^{J}, \quad\left|n_{1}\right| \simeq 2^{j_{1}}, \quad\left|m_{1}\right| \simeq 2^{\ell_{1}}
$$

in the inner sum enforces one of the following possibilities:

$$
\begin{aligned}
& \text { (A) } n_{1} \simeq m_{1} \text { and } J<\ell_{1}=j_{1} \text {, } \\
& \text { (B) } J=\ell_{1}>j_{1}, \\
& \text { (C) } J=j_{1}>\ell_{1},
\end{aligned}
$$

However $(\mathrm{A})$ is excluded by the condition $J \geqslant \min \left(\ell_{1}, j_{1}\right)$ in the outer sum.

- Case (B). Noting that in this case we have

$$
\frac{\left|m_{1}\right|^{2 s}}{m_{1}-n_{1}} \lesssim 2^{(2 s-1) \ell_{1}}
$$

Since the sum is restricted over $n_{1}+n_{2}=m_{1}+m_{2}$ we can assume that at leas one between $n_{2}, m_{2}$ is comparable to $m_{1}$. To fix the notations we will assume this index to be $n_{2}$, so that

$$
2^{\ell_{1}} \simeq\left|m_{1}\right| \simeq 2^{j_{2}} \simeq\left|n_{2}\right| \gtrsim\left|n_{1}\right|,\left|m_{2}\right|
$$


We have

$$
\begin{aligned}
& \left.(6.29)\right|_{(B)} \lesssim \sum_{\substack{j_{1}, j_{2}, \ell_{1}, \ell_{2} \in \mathbb{N} \\
\ell_{1}>j_{1}}} 2^{(2 s-1) \ell_{1}} \sum_{\substack{\left|n_{1}\right|,\left|n_{2}\right|,\left|m_{1}\right|,\left|m_{2}\right| \leqslant N \\
\left|m_{1}\right| \simeq 2^{\ell_{1}},\left|n_{1}\right| \simeq 2^{j_{1}} \\
\left|n_{2}\right| \simeq 2^{j_{2}},\left|m_{2}\right| \simeq 2^{\ell_{2}} \\
n_{1}+n_{2}=m_{1}+m_{2}}}\left|u\left(m_{1}\right)\right|\left|u\left(m_{2}\right)\right|\left|u\left(n_{1}\right)\right|\left|u\left(n_{2}\right)\right| \\
& \lesssim \sum_{j_{1}, j_{2}, \ell_{1}, \ell_{2} \in \mathbb{N}} 2^{\ell_{1}\left(s-\frac{1}{2}\right)} 2^{j_{2}\left(s-\frac{1}{2}\right)} \sum_{\substack{\left|n_{1}\right|,\left|n_{2}\right|,\left|m_{1}\right|,\left|m_{2}\right| \leqslant N \\
\left|m_{1}\right| \simeq 2^{\ell_{1}},\left|n_{1}\right| \simeq 2^{j_{1}} \\
\left|n_{2}\right| \simeq 2^{j_{2}},\left|m_{2}\right| \simeq 2^{\ell_{2}} \\
n_{1}+n_{2}=m_{1}+m_{2}}}\left|u\left(m_{1}\right)\right|\left|u\left(m_{2}\right)\right|\left|u\left(n_{1}\right)\right|\left|u\left(n_{2}\right)\right|
\end{aligned}
$$

Now for fixed $n_{1}, m_{2}$ we have, by Cauchy-Schwarz and Plancherel inequality

$$
2^{\ell_{1}\left(s-\frac{1}{2}\right)} 2^{j_{2}\left(s-\frac{1}{2}\right)} \sum_{\substack{\left|n_{2}\right|,\left|m_{1}\right| \leqslant N \\\left|m_{1}\right| \simeq 2^{\ell_{1}},\left|n_{2}\right| \simeq 2^{j_{2}} \\ n_{1}+n_{2}=m_{1}+m_{2}}}\left|u\left(m_{1}\right)\right|\left|u\left(n_{2}\right)\right| \leqslant 2^{\ell_{1}\left(s-\frac{1}{2}\right)}\left\|\Delta_{\ell_{1}} P_{N} u\right\|_{L^{2}} 2^{j_{2}\left(s-\frac{1}{2}\right)}\left\|\Delta_{j_{2}} P_{N} u\right\|_{L^{2}}
$$

Plugging this into the r.h.s. of (6.31) we get

$$
\begin{aligned}
&\left.(6.29)\right|_{(B)} \lesssim\left(\sum_{\ell_{1} \in \mathbb{N}} 2^{\ell_{1}\left(s-\frac{1}{2}\right)}\left\|\Delta_{\ell_{1}} P_{N} u\right\|_{L^{2}}\right)\left(\sum_{j_{2} \in \mathbb{N}} 2^{j_{2}\left(s-\frac{1}{2}\right)}\left\|\Delta_{j_{2}} P_{N} u\right\|_{L^{2}}\right) \\
& \times \sum_{j_{1}, \ell_{2} \in \mathbb{N}} \sum_{\substack{\left|n_{1}\right|,\left|m_{2}\right| \leqslant N \\
\left|n_{1}\right| \simeq 2^{j_{1}},\left|m_{2}\right| \simeq 2^{\ell_{2}}}}\left|u\left(m_{2}\right) \| u\left(n_{1}\right)\right| \\
& \lesssim X_{N}^{2}\left(\sum_{j_{1} \in \mathbb{N}} \sum_{\left|n_{1}\right| \simeq 2^{j_{1}}}\left|u\left(n_{1}\right)\right|\right)\left(\sum_{\ell_{2} \in \mathbb{N}} \sum_{\left|m_{2}\right| \simeq 2^{\ell_{2}}}\left|u\left(m_{2}\right)\right|\right)=X_{N}^{2} Y_{N}^{2}
\end{aligned}
$$

- Case (C). Since $\ell_{1}<j_{1}$ we have

$$
2^{(2 s-1) \ell_{1}} \leqslant 2^{\left(s-\frac{1}{2}\right) \ell_{1}} 2^{\left(s-\frac{1}{2}\right) j_{1}}
$$

thus

$$
\left.(6.29)\right|_{(C)} \lesssim \sum_{j_{1}, j_{2}, \ell_{1}, \ell_{2} \in \mathbb{N}} 2^{\left(s-\frac{1}{2}\right) \ell_{1}} 2^{\left(s-\frac{1}{2}\right) j_{1}} \sum_{\substack{\left|n_{1}\right|,\left|n_{2}\right|,\left|m_{1}\right|,\left|m_{2}\right| \leqslant N \\\left|m_{1}\right| \simeq 2^{\ell_{1}},\left|n_{1}\right| \simeq 2^{j_{1}} \\\left|n_{2}\right| \simeq 2^{j_{2}},\left|m_{2}\right| \simeq 2^{\ell_{2}} \\ n_{1}+n_{2}=m_{1}+m_{2}}}\left|u\left(m_{1}\right)\right|\left|u\left(m_{2}\right)\right|\left|u\left(n_{1}\right)\right|\left|u\left(n_{2}\right)\right|
$$

and proceed as in the case (B), switching $n_{1} \leftrightarrow n_{2}$.

It remains to bound

$$
\left|F_{N}^{<}\right|=\left|\operatorname{Im}\left(\sum_{\substack{j_{1}, j_{2}, \ell_{1}, \ell_{2} \in \mathbb{N} \\ J<\min \left(\ell_{1}, j_{1}\right)}} \sum_{\substack{\left|n_{1}\right|,\left|n_{2}\right|,\left|m_{1}\right|,\left|m_{2}\right| \leqslant N \\\left|m_{1}\right| \simeq 2^{\ell_{1}},\left|n_{1}\right| \simeq 2^{j_{1}} \\\left|n_{2}\right| \simeq 2^{j_{2}},\left|m_{2}\right| \simeq 2^{\ell_{2}} \\ 0 \neq\left|n_{1}-m_{1}\right| \simeq 2^{J} \\ n_{1}+n_{2}=m_{1}+m_{2}}} \frac{\left|m_{1}\right|^{2 s}}{m_{1}-n_{1}} \bar{u}\left(m_{1}\right) \bar{u}\left(m_{2}\right) u\left(n_{1}\right) u\left(n_{2}\right)\right)\right| .
$$


Since $J<\min \left(\ell_{1}, j_{1}\right)$ we are summing over $n_{1} \neq 0$. Using the fractional binomial identity (recall $\left.n_{2}-m_{2}=m_{1}-n_{1}\right)$

$$
\begin{aligned}
\frac{\left|m_{1}\right|^{2 s}}{m_{1}-n_{1}} & =\frac{\left|m_{1}\right|^{s}\left|n_{1}+n_{2}-m_{2}\right|^{s}}{m_{1}-n_{1}} \\
& =\frac{\left|m_{1}\right|^{s}\left|n_{1}\right|^{s}\left(1+\frac{n_{2}-m_{2}}{\left|n_{1}\right|}\right)^{s}}{m_{1}-n_{1}}=\sum_{k \geqslant 0} \frac{(s)_{k}}{k !} \frac{\left|m_{1}\right|^{s}\left|n_{1}\right|^{s}\left(m_{1}-n_{1}\right)^{k-1}}{\left|n_{1}\right|^{k}}
\end{aligned}
$$

and the cancellation already exploited in Lemma 5.1 (see formula (5.11))

$$
\operatorname{Im}\left(\sum_{\substack{j_{1}, j_{2}, \ell_{1}, \ell_{2} \in \mathbb{N} \\ J<\min \left(\ell_{1}, j_{1}\right)}} \sum_{\substack{\left|n_{1}\right|,\left|n_{2}\right|,\left|m_{1}\right|,\left|m_{2}\right| \leqslant N \\\left|m_{1}\right| \simeq 2^{\ell_{1}},\left|n_{1}\right| \simeq 2^{j_{1}} \\\left|n_{2}\right| \simeq 2^{j_{2}},\left|m_{2}\right| \simeq 2^{\ell_{2}} \\ 0 \neq\left|n_{1}-m_{1}\right| \simeq 2^{J} \\ n_{1}+n_{2}=m_{1}+m_{2}}} \frac{\left|m_{1}\right|^{s}\left|n_{1}\right|^{s}}{m_{1}-n_{1}} \bar{u}\left(m_{1}\right) \bar{u}\left(m_{2}\right) u\left(n_{1}\right) u\left(n_{2}\right)\right)=0,
$$

we arrive to

$$
\left|F_{N}^{<}\right| \lesssim\left|\sum_{k \geqslant 1} \frac{(s)_{k}}{k !} \operatorname{Im}\left(\sum_{\substack{j_{1}, j_{2}, \ell_{1}, \ell_{2} \in \mathbb{N} \\ J<\min \left(\ell_{1}, j_{1}\right)}} \sum_{\substack{\left|n_{1}\right|,\left|n_{2}\right|,\left|m_{1}\right|,\left|m_{2}\right| \leqslant N \\\left|m_{1}\right| \simeq 2^{\ell_{1}},\left|n_{1}\right| \simeq 2^{j_{1}} \\\left|n_{2}\right| \simeq 2^{j_{2}},\left|m_{2}\right| \simeq 2^{\ell_{2}} \\ 0 \neq\left|n_{1}-m_{1}\right| \simeq 2^{J} \\ n_{1}+n_{2}=m_{1}+m_{2}}} \frac{\left|m_{1}\right|^{s}\left|n_{1}\right|^{s}\left(m_{1}-n_{1}\right)^{k-1}}{\left|n_{1}\right|^{k}} \bar{u}\left(m_{1}\right) \bar{u}\left(m_{2}\right) u\left(n_{1}\right) u\left(n_{2}\right)\right)\right| .
$$

Since $J<\min \left(\ell_{1}, j_{1}\right)$ implies $\left|n_{1}-m_{1}\right| \leqslant \frac{1}{2}\left|n_{1}\right|$ and that $n_{1}$ and $m_{1}$ are comparable, bringing the modulus inside and summing over $k$ we arrive to

$$
\left|F_{N}^{<}\right| \lesssim \sum_{j_{1}, j_{2}, \ell_{1}, \ell_{2} \in \mathbb{N}} 2^{\left(s-\frac{1}{2}\right) \ell_{1}} 2^{\left(s-\frac{1}{2}\right) j_{2}} \sum_{\substack{\left|n_{1}\right|,\left|n_{2}\right|,\left|m_{1}\right|,\left|m_{2}\right| \leqslant N \\\left|m_{1}\right| \simeq 2^{\ell_{1}},\left|n_{1}\right| \simeq 2^{j_{1}} \\\left|n_{2}\right| \simeq 2^{j_{2}},\left|m_{2}\right| \simeq 2^{\ell_{2}} \\ n_{1}+n_{2}=m_{1}+m_{2}}}\left|u\left(m_{1}\right)\right|\left|u\left(m_{2}\right)\right|\left|u\left(n_{1}\right)\right|\left|u\left(n_{2}\right)\right|
$$

and we proceed as in the case (B).

\section{REFERENCES}

[1] H. Bahouri and G. Perelman, Global well-posedness for the derivative nonlinear Schrödinger equation, arXiv: 2012.01923v1.

[2] J. Bourgain, Periodic nonlinear Schrödinger equation and invariant measures, Commun. Math. Phys. 166 (1994), 1-26.

[3] J. Brereton, Invariant measure construction at a fixed mass, Nonlinearity 32 (2019), 496-558.

[4] R. Cameron, W. Martin, Transformations of Wiener Integrals under Translations. Annals of Mathematics. 45 (1944), 386-396.

[5] A. B. Cruzeiro, Equations différentielles ordinaires: non- explosion et measures quasi invariantes, J. Funct. Anal. 54 (1983), 193-205.

[6] A. B. Cruzeiro, Equations différentielles sur l'espace de Wiener et formules de Cameron-Martin non linéaires, J. Funct. Anal. 54 (1983), 206-227.

[7] A. Debussche, Y. Tsustumi, Quasi-Invariance of Gaussian Measures Transported by the Cubic NLS with ThirdOrder Dispersion on $\mathbb{T}$, arXiv:2002.04899 (2020).

[8] Y. Deng, A. Nahmod, H. Yue, Optimal local well-posedness for the periodic derivative nonlinear Schrödinger equation arXiv:1905.04352 (2019).

[9] Y. Deng, N. Tzvetkov, N. Visciglia, Invariant measures and long time behaviour for the Benjamin-Ono equation III, Comm. Math. Phys. 339 (2015), 815-857.

[10] J. Forlano, W. Trenberth, On the transport of Gaussian measures under the one-dimensional fractional nonlinear Schrödinger equation, Ann. Inst. Henri Poincare, Anal. Non Lineaire, 36 (2019), 1987-2025.

[11] G. Genovese, R. Lucà, D. Valeri, Gibbs measures associated to the integrals of motion of the periodic derivative nonlinear Schrödinger equation, Sel. Math. New Ser. 22 (2016), 1663-1702.

[12] G. Genovese, R. Lucà, D. Valeri, Invariant Measures for the periodic derivative nonlinear Schroedinger equation, Math. Annalen, 374 (2019),1075-1138. 
[13] I. Girsanov, On transforming a certain class of stochastic processes by absolutely continuous substitution of measures. Theory of Probability and its Applications. 5 (1960) 285-301.

[14] T. S. Gunaratnam, T. Oh, N. Tzvetkov, H. Weber, Quasi-invariant Gaussian measures for the nonlinear wave equation in three dimensions, arXiv:1808.03158 (2018).

[15] A. Grünrock, S. Herr, Low regularity local well-posedness of the derivative nonlinear Schrödinger equation with periodic initial data, SIAM J. Math. Anal. 39 (2008), 1890-1920.

[16] S. Herr, On the Cauchy problem for the Derivative nonlinear Schrödinger equation with periodic boundary condition, Int. Math. Res. Notices (2006).

[17] R. Jenkins, J. Liu, P. Perry, C. Sulem, Global Existence for the Derivative Nonlinear Schrödinger Equation with Arbitrary Spectral Singularities, arXiv:1804.01506 [math.AP].

[18] H.-H. Kuo, Integration theory on infinite-dimensional manifolds, Trans. Amer. Math. Soc., 159 (1971), 57-78.

[19] H.-H. Kuo, Gaussian Measures in Banach Spaces, Springer-Verlag (1975).

[20] J.-H. Lee, Global solvability of the derivative nonlinear Schrödinger equation, Trans. Amer. Math. Soc., 314 (1989), 107-118.

[21] P. Malliavin, Stochastic Analysis, Springer (1997).

[22] A. Nahmod, T. Oh, L. Rey-Bellet, G. Staffilani, Invariant weighted Wiener measures and almost sure global well-posedness for the periodic derivative NLS, J. Eur. Math. Soc. (JEMS) 14 (2012), 1275-1330.

[23] A. Nahmod, L. Rey-Bellet, S. Sheffield, G. Staffilani, Absolute continuity of Brownian bridges under certain gauge transformations, Math. Res. Letters 18 (2011), 875-887.

[24] T. Oh, N. Tzvetkov, Quasi-invariant Gaussian measures for the cubic fourth order nonlinear Schrödinger equation, Probab. Theory Relat. Fields 169 (2017), 1121-1168.

[25] T. Oh, N. Tzvetkov, Quasi-invariant Gaussian measures for the two-dimensional defocusing cubic nonlinear wave equation, to appear on JEMS .

[26] T. Oh, P. Sosoe, N. Tzvetkov, An optimal regularity result on the quasi-invariant Gaussian measures for the cubic fourth order nonlinear Schrödinger equation, J. Ec. Polytechnique, Math., 5 (2018), 793-841.

[27] T. Oh, Y. Tsutsumi and N. Tzvetkov, Quasi-invariant Gaussian measures for the cubic nonlinear Schroedinger equation with third-order dispersion, C. R. Acad. Sci. Paris, Ser. I, 357 (2019), 366-381.

[28] F. Planchon, N. Tzvetkov and N. Visciglia, Transport of Gaussian measures by the flow of the nonlinear Schrödinger equation, Math. Ann. 378 (2020) 389-423.

[29] R. Ramer, On nonlinear transformations of Gaussian measures, Journal of Functional Analysis 15 (1974), 166-187.

[30] P. Sosoe, W. J. Trenberth and T. Xiao, Quasi-invariance of fractional Gaussian fields by nonlinear wave equation with polynomial nonlinearity, arXiv:1906.02257v1 (2019).

[31] L. Thomann, N. Tzvetkov, Gibbs measure for the periodic derivative nonlinear Schrödinger equation, Nonlinearity 23 (2010), 2771-2791.

[32] N. Tzvetkov, Construction of a Gibbs measure associated to the periodic Benjamin-Ono equation, Probability theory and related fields 146 (2010), 481-514.

[33] N. Tzvetkov, Quasi-invariant Gaussian measures for one dimensional Hamiltonian PDEs, Forum Math. Sigma 3 (2015), e28, 35 pp.

[34] N. Tzvetkov, N. Visciglia, Gaussian measures associated to the higher order conservation laws of the BenjaminOno equation, Ann. Sci. ENS 46 (2013) 249-299 (2013).

[35] N. Tzvetkov, N. Visciglia, Invariant measures and long time behaviour for the Benjamin-Ono equation, Int. Math. Res. Not. 17 (2014) 4679-4614.

[36] N. Tzvetkov, N. Visciglia, Invariant measures and long time behaviour for the Benjamin-Ono equation II, J. Math. Pures Appl. 103 (2015), 102-141.

[37] A.S. Üstünel, M. Zakai, Transformation of measure on Wiener space, Springer Science \& Business Media, (2013).

Department Mathematik und Informatik, Universität Basel Spiegelgasse 1, CH-4051 Basel, SwitzerLAND

Email address: giuseppe.genovese@unibas.ch

BCAM - Basque Center for Applied Mathematics, 48009 Bilbao, Spain and Ikerbasque, Basque Foundation for Science, 48011 Bilbao, Spain.

Email address: rluca@bcamath.org

Department of Mathematics (AGM), University of Cergy-Pontoise, 2, av. Adolphe Chauvin, 95302 Cergy-Pontoise Cedex, FRAnCE

Email address: nikolay.tzvetkov@u-cergy.fr 